\title{
THE AVERAGING OF NONLOCAL HAMILTONIAN STRUCTURES IN WHITHAM'S METHOD
}

\author{
ANDREI YA. MALTSEV
}

Received 16 June 2001

\begin{abstract}
We consider the $m$-phase Whitham's averaging method and propose the procedure of "averaging" nonlocal Hamiltonian structures. The procedure is based on the existence of a sufficient number of local-commuting integrals of the system and gives the Poisson bracket of Ferapontov type for Whitham's system. The method can be considered as the generalization of the Dubrovin-Novikov procedure for the local field-theoretical brackets.
\end{abstract}

2000 Mathematics Subject Classification: 70K70, 70S05, 70S10, 70K65, 70G75, 70K43.

1. Introduction. We consider the averaging of the nonlocal Hamiltonian structures in Whitham's averaging method. As it is well known, Whitham's method permits to obtain the equations on the "slow" modulated parameters of the exact periodic or quasi-periodic solutions of systems of partial differential equations and it was pointed out by Whitham [32] that these equations inherit the local Lagrangian structure if the initial system has it. The Lagrangian formalism for Whitham's system is given in this approach by "averaging" the local Lagrangian function for the initial system on the corresponding space of (quasi)-periodic solutions. Some basic questions concerning Whitham's method can be found in [4, 5, 6, 7, 17, 19, 20, 32].

Dubrovin and Novikov also investigated the question of the conservation of local field-theoretical Hamiltonian structures in Whitham's method and suggested the procedure of "averaging" of local field-theoretical Poisson bracket to obtain the Poisson bracket of Hydrodynamic type for Whitham's system (see [5, 6, 7, 28]).

The Jacobi identity for the averaged bracket and the invariance of the DubrovinNovikov procedure of averaging was proved by the author in [23] (see also [21]) using the Dirac restriction procedure of the initial bracket on the subspace of quasi-periodic " $m$-phase" solutions of the initial system. The connection between the procedure of Dubrovin and Novikov, and the procedure of averaging of the Lagrangian function in the case when the initial local Hamiltonian structure just follows from the local Lagrangian one, can be found in [25].

Some extension of the averaging "local" Hamiltonian structures for the case of discrete systems is also presented in [22].

In the present work, we deal with the Poisson brackets having the nonlocal part of the form

$$
\begin{aligned}
\left\{\varphi^{i}(x), \varphi^{j}(y)\right\}= & \sum_{k \geq 0} B_{k}^{i j}\left(\varphi, \varphi_{x}, \ldots\right) \delta^{(k)}(x-y) \\
& +\sum_{k \geq 0} e_{k} S_{(k)}^{i}\left(\varphi, \varphi_{x}, \ldots\right) v(x-y) S_{(k)}^{j}\left(\varphi, \varphi_{y}, \ldots\right),
\end{aligned}
$$


where $e_{k}= \pm 1, v(x-y)=-v(y-x), \partial_{x} v(x-y)=\delta(x-y)$, and both sums contain the finite number of terms depending on the finite number of derivatives of $\varphi$ with respect to $x$.

We point out here that the brackets (1.1) can be found in the so-called "integrable" systems (see [8, 24, 30]).

The most general form of the nonlocal Hamiltonian operators (1.1) containing only $\delta^{\prime}(X-Y)$ and $\delta(X-Y)$ in the local part and the quasi-linear fluxes $S_{(k) j}^{i}(U) U_{X}^{j}$ of "hydrodynamic" type in the nonlocal one, was suggested by Ferapontov in [9] as the generalization of the bracket introduced in [26]. We discuss here the possibility of "averaging" the brackets (1.1) in Whitham's method to obtain the bracket of such "Hydrodynamic type" for Whitham's system.

As was shown by Ferapontov, the Hamiltonian operators of this type reveal a beautiful differential-geometrical structure following from the Jacobi identity of the bracket (see $[9,10,11,16])$. In particular, they can be obtained as the Dirac restriction of local differential-geometrical Poisson brackets on the space with flat normal connection [11].

The first example of the nonlocal bracket (of Mokhov-Ferapontov type, see [26]) for Whitham's system, for NS equation in the one-phase case, was constructed by Pavlov in [29] from a nice differential-geometrical consideration. After that there was a question about the possibility of constructing the nonlocal Hamiltonian structures for Whitham's system from the structures (1.1) for the initial one. As was mentioned above, the Hamiltonian operators (1.1) exist for many "integrable" systems like KdV and in [16] (see also [2]) there was a discussion of the possibility of averaging the nonlocal operators for KdV equation using the local bi-Hamiltonian structure and the recursion operator for the two averaged local Poisson brackets. The corresponding calculations for the $m$-phase periodic solutions of KdV were made by Alekseev in [1].

Here we propose the general construction for the averaging of operators (1.1) in Whitham's method which is the generalization of the Dubrovin-Novikov procedure for the case of the presence of nonlocal terms in the bracket. Our procedure does not require the local bi-Hamiltonian structure and can be used in the general situation. As in the procedure of Dubrovin and Novikov, we require here the existence of sufficient number of local-commuting integrals generating the local flows according to (1.1). We also impose the conditions of "regularity" of the full family of $m$-phase solutions as in the local case (see [23]).

2. Some general properties of nonlocal brackets. Consider the nonlocal 1-dimensional Hamiltonian structure of the type

$$
\begin{aligned}
\left\{\varphi^{i}(x), \varphi^{j}(y)\right\}= & \sum_{k \geq 0} B_{(k)}^{i j}\left(\varphi, \varphi_{x}, \ldots\right) \delta^{(k)}(x-y) \\
& +\sum_{k \geq 0} \tilde{S}_{(k)}^{i}\left(\varphi, \varphi_{x}, \ldots\right) v(x-y) \tilde{T}_{(k)}^{j}\left(\varphi, \varphi_{y}, \ldots\right), \quad 1 \leq i, j \leq n,
\end{aligned}
$$

where we have the finite number of terms in both sums depending on the finite number of derivatives of $\varphi$ with respect to $x$. 
We call the local translation invariant Hamiltonian function, the functional of the form

$$
H[\varphi]=\int \mathscr{P}_{H}\left(\varphi, \varphi_{x}, \ldots\right) d x
$$

Here $v(x-y)$ is the skew-symmetric function such that

$$
D_{x} v(x-y)=\delta(x-y)
$$

and $\delta^{(k)}(x-y)$ is the $k$ th derivative of the delta-function with respect to $x$.

We assume that the bracket (2.1) is written in the "irreducible" form, that is, the number of terms in the second sum is the minimal possible, and the sets $\tilde{S}_{(k)}^{i}$ and $\tilde{T}_{(k)}^{j}$ are both linearly independent. From the skew-symmetry of the bracket (2.1), it follows that the sets of $\tilde{S}_{(k)}^{i}$ and $\tilde{T}_{(k)}^{j}$ coincide and it can be easily seen that the bracket (2.1) can be represented in the "canonical" form (1.1).

Indeed since the sets $\left\{\tilde{S}_{(k)}^{i}\right\}$ and $\left\{\tilde{T}_{k}^{j}\right\}$ coincide, we have the one finite-dimensional linear space generated by fluxes (vector fields)

$$
\varphi_{\tau_{k}}^{i}=\tilde{S}_{(k)}^{i}\left(\varphi, \varphi_{x}, \ldots\right)
$$

and the symmetric (view the skew-symmetry of the bracket and the function $v(x-y)$ ) finite-dimensional constant 2-form which describes their couplings in the nonlocal part of (2.1). So, we can write it in the canonical form according to its signature after some linear transformation of the flows $\hat{\tilde{S}}_{(k)}$ with constant coefficients.

We should also define in every case the functional space where we consider the action of the Hamiltonian operator (1.1) and this can depend on the concrete situation. The most natural thing is to consider the functional space $\varphi(x)$ and the algebra of functionals $I[\varphi]$ such that their variational derivatives multiplied by the flows $S_{(k)}\left(\varphi, \varphi_{x}, \ldots\right)$ give us the rapidly decreasing functions as $|x| \rightarrow \infty$. Here we use the functionals of the type

$$
\int \sum_{p=1}^{n} \varphi^{p}(x) q_{p}(x) d x
$$

where $q_{p}(x)$ are arbitrary smooth functions with compact supports. To get all the properties of the bracket (1.1) and for the other functionals used in the considerations, we assume that they have the form compatible with the bracket (1.1) in the sense discussed above.

We construct the procedure which gives us the brackets of Ferapontov type [9, 10, $11,16]$

$$
\begin{aligned}
\left\{U^{\nu}(X), U^{\mu}(Y)\right\}= & g^{v \mu}(U) \delta^{\prime}(X-Y)+b_{\lambda}^{v \mu}(U) U_{X}^{\lambda} \delta(X-Y) \\
& +\sum_{k \geq 0} e_{k} S_{(k) \lambda}^{v}(U) U_{X}^{\lambda} v(X-Y) S_{(k) \delta}^{\mu}(U) U_{Y}^{\delta}, \quad 1 \leq \nu, \mu, \lambda, \delta \leq N
\end{aligned}
$$


from the initial bracket (1.1) of the general form after the averaging on the appropriate family of exact $m$-phase solutions.

So for the Poisson brackets (1.1), we consider Whitham's method for the local fluxes generated by Hamiltonian functions (2.2) (if they exist), that is,

$$
\varphi_{t}^{i}=Q^{i}\left(\varphi, \varphi_{x}, \ldots\right)
$$

Now we formulate some general theorems about the nonlocal part of the bracket (1.1).

THEOREM 2.1. For any nonlocal Hamiltonian operator written in the "canonical" form (1.1),

(1) the flows

$$
\dot{\varphi}^{i}=S_{(k)}^{i}\left(\varphi, \varphi_{x}, \ldots\right)
$$

commute with each other;

(2) any of the flows (2.8) conserves the Hamiltonian structure (2.1).

PROoF. Consider the functional (2.5) for some $q_{p}(x)$ with the compact supports and consider the Hamiltonian flow $\xi^{i}(x)$ generated by (2.5) according to (1.1), that is,

$$
\begin{aligned}
\xi^{i}(x)= & \sum_{k \geq 0} B_{k}^{i j}\left(\varphi, \varphi_{x}, \ldots\right) \frac{d^{k}}{d x^{k}} q_{j}(x) \\
& +\frac{1}{2} \sum_{k \geq 0} e_{k} S_{(k)}^{i}\left(\varphi, \varphi_{x}, \ldots\right) \\
& \times\left[\int_{-\infty}^{x} S_{(k)}^{j}\left(\varphi, \varphi_{z}, \ldots\right) q_{j}(z) d z-\int_{x}^{\infty} S_{(k)}^{j}\left(\varphi, \varphi_{z}, \ldots\right) q_{j}(z) d z\right],
\end{aligned}
$$

where $e_{k}= \pm 1$ (there is a summation over the repeated indices).

For the Hamiltonian flow $\xi^{i}(x)$, we should have

$$
\left[\mathscr{L}_{\xi} \hat{J}\right]^{i j}(x, y) \equiv 0
$$

where $\hat{J}$ is the Hamiltonian operator $(1.1)$ and $\mathscr{L}_{\xi}$ is the Lie-derivative given by the expression

$$
\begin{aligned}
{\left[\mathscr{L}_{\xi} \hat{J}\right]^{i j}(x, y)=} & \int \xi^{s}(z) \frac{\delta}{\delta \varphi^{s}(z)} J^{i j}(x, y) d z \\
& -\int J^{s j}(z, y) \frac{\delta}{\delta \varphi^{s}(z)} \xi^{i}(x) d z \\
& -\int J^{i s}(x, z) \frac{\delta}{\delta \varphi^{s}(z)} \xi^{j}(y) d z .
\end{aligned}
$$


Consider the relation (2.10) for $x$ and $y$ larger than any $z$ from the support of $q_{p}(z)$. Then we have

$$
\begin{aligned}
& {\left[\mathscr{L}_{\xi} \hat{J}\right]^{i j}(x, y)=\sum_{k \geq 0} \dot{B}_{k}^{i j}\left(\varphi, \varphi_{x}, \ldots\right) \delta^{(k)}(x-y)} \\
& +\sum_{k \geq 0} e_{k} \dot{S}_{(k)}^{i}\left(\varphi, \varphi_{x}, \ldots\right) v(x-y) S_{(k)}^{j}\left(\varphi, \varphi_{y}, \ldots\right) \\
& +\sum_{k \geq 0} e_{k} S_{(k)}^{i}\left(\varphi, \varphi_{x}, \ldots\right) v(x-y) \dot{S}_{(k)}^{j}\left(\varphi, \varphi_{y}, \ldots\right) \\
& -\sum_{k \geq 0}(-1)^{k} \frac{d^{k}}{d y^{k}}\left(B_{k}^{s j}\left(\varphi, \varphi_{y}, \ldots\right) \sum_{k^{\prime} \geq 0} e_{k^{\prime}} \frac{\delta S_{\left(k^{\prime}\right)}^{i}\left(\varphi, \varphi_{x}, \ldots\right)}{\delta \varphi^{s}(y)}\right) \\
& \times\left[\frac{1}{2} \int_{-\infty}^{\infty} S_{\left(k^{\prime}\right)}^{p}\left(\varphi, \varphi_{w}, \ldots\right) q_{p}(w) d w\right] \\
& -\sum_{k \geq 0} B_{k}^{i s}\left(\varphi, \varphi_{x}, \ldots\right) \frac{d^{k}}{d x^{k}}\left(\sum_{k^{\prime} \geq 0} e_{k^{\prime}} \frac{\delta S_{\left(k^{\prime}\right)}^{j}\left(\varphi, \varphi_{y}, \ldots\right)}{\delta \varphi^{s}(x)}\right) \\
& \times\left[\frac{1}{2} \int_{-\infty}^{\infty} S_{\left(k^{\prime}\right)}^{p}\left(\varphi, \varphi_{w}, \ldots\right) q_{p}(w) d w\right] \\
& -\int d z \sum_{k \geq 0} e_{k} S_{(k)}^{s}\left(\varphi, \varphi_{z}, \ldots\right) v(z-y) S_{(k)}^{j}\left(\varphi, \varphi_{y}, \ldots\right) \\
& \times \sum_{k^{\prime} \geq 0} e_{k^{\prime}} \frac{\delta}{\delta \varphi^{s}(z)}\left(S_{\left(k^{\prime}\right)}^{i}\left(\varphi, \varphi_{x}, \ldots\right)\left[\frac{1}{2} \int_{-\infty}^{\infty} S_{\left(k^{\prime}\right)}^{p}\left(\varphi, \varphi_{w}, \ldots\right) q_{p}(w) d w\right]\right) \\
& -\int d z \sum_{k \geq 0} e_{k} S_{(k)}^{i}\left(\varphi, \varphi_{x}, \ldots\right) v(x-z) S_{(k)}^{s}\left(\varphi, \varphi_{z}, \ldots\right) \\
& \times \sum_{k^{\prime} \geq 0} e_{k^{\prime}} \frac{\delta}{\delta \varphi^{s}(z)}\left(S_{\left(k^{\prime}\right)}^{j}\left(\varphi, \varphi_{y}, \ldots\right)\left[\frac{1}{2} \int_{-\infty}^{\infty} S_{\left(k^{\prime}\right)}^{p}\left(\varphi, \varphi_{w}, \ldots\right) q_{p}(w) d w\right]\right),
\end{aligned}
$$

where $\dot{B}_{k}^{i j}\left(\varphi, \varphi_{x}, \ldots\right)$ and $\dot{S}_{(k)}^{i}\left(\varphi, \varphi_{x}, \ldots\right)$ are the derivatives of these functions with respect to the flow

$$
\varphi_{t}^{i}=\sum_{k \geq 0} e_{k} S_{(k)}^{i}\left(\varphi, \varphi_{x}, \ldots\right)\left[\frac{1}{2} \int_{-\infty}^{\infty} S_{(k)}^{p}\left(\varphi, \varphi_{w}, \ldots\right) q_{p}(w) d w\right]
$$

Here we used that $x, y>\operatorname{supp} q_{p}$ in the expression (2.9) for $\xi^{i}(x)$ and $\xi^{j}(y)$, and also omitted the variational derivatives, with respect to $\varphi^{s}(x)$ and $\varphi^{s}(y)$, of the nonlocal expressions containing the convolutions with $q_{p}(w)$. 
So, we have

$$
\begin{aligned}
& 0 \equiv\left[\mathscr{L}_{\xi} \hat{J}\right]^{i j}(x, y) \\
& =\sum_{k \geq 0} \dot{B}_{k}^{i j}\left(\varphi, \varphi_{x}, \ldots\right) \delta^{(k)}(x-y)+\sum_{k \geq 0} e_{k} \dot{S}_{(k)}^{i}\left(\varphi, \varphi_{x}, \ldots\right) \nu(x-y) S_{(k)}^{j}\left(\varphi, \varphi_{y}, \ldots\right) \\
& +\sum_{k \geq 0} e_{k} S_{(k)}^{i}\left(\varphi, \varphi_{x}, \ldots\right) v(x-y) \dot{S}_{(k)}^{j}\left(\varphi, \varphi_{y}, \ldots\right) \\
& -\sum_{k \geq 0}(-1)^{k} \frac{d^{k}}{d y^{k}}\left(B_{k}^{s j}\left(\varphi, \varphi_{y}, \ldots\right) \sum_{k^{\prime} \geq 0} e_{k^{\prime}} \frac{\delta S_{\left(k^{\prime}\right)}^{i}\left(\varphi, \varphi_{x}, \ldots\right)}{\delta \varphi^{s}(y)}\right) \\
& \times\left[\frac{1}{2} \int_{-\infty}^{\infty} S_{\left(k^{\prime}\right)}^{p}\left(\varphi, \varphi_{w}, \ldots\right) q_{p}(w) d w\right] \\
& -\sum_{k \geq 0} B_{k}^{i s}\left(\varphi, \varphi_{x}, \ldots\right) \frac{d^{k}}{d x^{k}}\left(\sum_{k^{\prime} \geq 0} e_{k^{\prime}} \frac{\delta S_{\left(k^{\prime}\right)}^{j}\left(\varphi, \varphi_{y}, \ldots\right)}{\delta \varphi^{s}(x)}\right) \\
& \times\left[\frac{1}{2} \int_{-\infty}^{\infty} S_{\left(k^{\prime}\right)}^{p}\left(\varphi, \varphi_{w}, \ldots\right) q_{p}(w) d w\right] \\
& -\int d z \sum_{k \geq 0} e_{k} S_{(k)}^{s}\left(\varphi, \varphi_{z}, \ldots\right) v(z-y) S_{(k)}^{j}\left(\varphi, \varphi_{y}, \ldots\right) \\
& \times \sum_{k^{\prime} \geq 0} e_{k^{\prime}} \frac{\delta S_{\left(k^{\prime}\right)}^{i}\left(\varphi, \varphi_{x}, \ldots\right)}{\delta \varphi^{s}(z)}\left[\frac{1}{2} \int_{-\infty}^{\infty} S_{\left(k^{\prime}\right)}^{p}\left(\varphi, \varphi_{w}, \ldots\right) q_{p}(w) d w\right] \\
& -\int d z \sum_{k \geq 0} e_{k} S_{(k)}^{i}\left(\varphi, \varphi_{x}, \ldots\right) v(x-z) S_{(k)}^{s}\left(\varphi, \varphi_{z}, \ldots\right) \\
& \times \sum_{k^{\prime} \geq 0} e_{k^{\prime}} \frac{\delta S_{\left(k^{\prime}\right)}^{j}\left(\varphi, \varphi_{y}, \ldots\right)}{\delta \varphi^{s}(z)}\left[\frac{1}{2} \int_{-\infty}^{\infty} S_{\left(k^{\prime}\right)}^{p}\left(\varphi, \varphi_{w}, \ldots\right) q_{p}(w) d w\right] \\
& -\int d z \sum_{k \geq 0} e_{k} S_{(k)}^{s}\left(\varphi, \varphi_{z}, \ldots\right) v(x-y) S_{(k)}^{j}\left(\varphi, \varphi_{y}, \ldots\right) \\
& \times \sum_{k^{\prime} \geq 0} e_{k^{\prime}} S_{\left(k^{\prime}\right)}^{i}\left(\varphi, \varphi_{x}, \ldots\right) \frac{1}{2}\left[\frac{\delta}{\delta \varphi^{s}(z)} \int_{-\infty}^{\infty} S_{\left(k^{\prime}\right)}^{p}\left(\varphi, \varphi_{w}, \ldots\right) q_{p}(w) d w\right] \\
& -\int d z \sum_{k \geq 0} e_{k} S_{(k)}^{i}\left(\varphi, \varphi_{x}, \ldots\right) v(x-y) S_{(k)}^{s}\left(\varphi, \varphi_{z}, \ldots\right) \\
& \times \sum_{k^{\prime} \geq 0} e_{k^{\prime}} S_{\left(k^{\prime}\right)}^{j}\left(\varphi, \varphi_{y}, \ldots\right) \frac{1}{2}\left[\frac{\delta}{\delta \varphi^{s}(z)} \int_{-\infty}^{\infty} S_{\left(k^{\prime}\right)}^{p}\left(\varphi, \varphi_{w}, \ldots\right) q_{p}(w) d w\right] \\
& \equiv \sum_{k \geq 0} e_{k}\left[\frac{1}{2} \int_{-\infty}^{\infty} S_{(k)}^{p}\left(\varphi, \varphi_{w}, \ldots\right) q_{p}(w) d w\right] \cdot\left[\mathscr{L}_{k} \hat{J}\right]^{i j}(x, y) \\
& +\sum_{k, k^{\prime} \geq 0} e_{k} e_{k^{\prime}} S_{\left(k^{\prime}\right)}^{i}\left(\varphi, \varphi_{x}, \ldots\right) S_{(k)}^{j}\left(\varphi, \varphi_{y}, \ldots\right)
\end{aligned}
$$




$$
\begin{aligned}
\times \frac{1}{2} \int( & S_{(k)}^{s}\left(\varphi, \varphi_{z}, \ldots\right) \frac{\delta}{\delta \varphi^{s}(z)}\left[\int_{-\infty}^{\infty} S_{\left(k^{\prime}\right)}^{p}\left(\varphi, \varphi_{w}, \ldots\right) q_{p}(w) d w\right] \\
& \left.-S_{\left(k^{\prime}\right)}^{s}\left(\varphi, \varphi_{z}, \ldots\right) \frac{\delta}{\delta \varphi^{s}(z)}\left[\int_{-\infty}^{\infty} S_{(k)}^{p}\left(\varphi, \varphi_{w}, \ldots\right) q_{p}(w) d w\right]\right) d z
\end{aligned}
$$

where $\left[\mathscr{L}_{k} \hat{J}\right]^{i j}(x, y)$ are the Lie derivatives of $\hat{J}$ with respect to the flows (2.8) $\dot{\varphi}^{i}=$ $S_{(k)}^{i}\left(\varphi, \varphi_{x}, \ldots\right)$.

As it can be easily seen, the last term in (2.14) is the only one containing the functions not equal to zero when $x \neq y$, which are not skew-symmetric as $x \rightarrow y$ (the other nonlocal terms contain the function $v(x-y)$ ). So from (2.14), we have that it should be identically zero for any $q_{p}(w)$ with the support satisfying the conditions described above $\left(x, y>\operatorname{supp} q_{p}(w)\right)$. Using the standard expression for the variational derivative and the integration by parts, we obtain that this term can be written in the form

$$
\sum_{k, k^{\prime} \geq 0} e_{k} e_{k^{\prime}} S_{\left(k^{\prime}\right)}^{i}\left(\varphi, \varphi_{x}, \ldots\right) S_{(k)}^{j}\left(\varphi, \varphi_{y}, \ldots\right) \times \frac{1}{2} \int_{-\infty}^{\infty} q_{p}(z)\left[S_{(k)}, S_{\left(k^{\prime}\right)}\right]^{p}(z) d z,
$$

for any $q_{p}(z)\left(x, y>\operatorname{supp} q_{p}(z)\right)$ where $\left[S_{(k)}, S_{\left(k^{\prime}\right)}\right]$ is the commutator of the flows (2.8). So for the linearly independent set of $S_{(k)}$, we obtain

$$
\left[S_{(k)}, S_{\left(k^{\prime}\right)}\right] \equiv 0 .
$$

From (2.14) we also have for the linearly independent set of $S_{(k)}$ and different $q_{p}(w)$ that

$$
\left[\mathscr{L}_{k} \hat{J}\right]^{i j}(x, y) \equiv 0
$$

So, Theorem 2.1 is proved.

It is also obvious that the statements of the theorem are valid for all the brackets (2.1) written in the "irreducible" form since all $\tilde{S}_{(k)}$ and $\tilde{T}_{(k)}$ in this case are just the linear combinations of the flows $S_{(k)}$.

REMARK 2.2. We point here that the first statement of the theorem for the diagonalizible nonlocal brackets (2.6) of Ferapontov type was proved previously by Ferapontov in [9] using the differential-geometrical considerations. In [9, 10, 11, 16] also the full classification of the brackets (2.6), from the differential geometrical point of view, can be found.

It is easy to see that the local functional of type (2.2)

$$
I=\int \mathscr{P}\left(\varphi, \varphi_{x}, \ldots\right) d x
$$

generates the local flow in the Hamiltonian structure (2.1) if and only if the derivative of its density $\mathscr{P}\left(\varphi, \varphi_{x}, \ldots\right)$, with respect to any of the flows (2.8), is the total derivative with respect to $x$, that is, there exist $\mathscr{2}_{(k)}\left(\varphi, \varphi_{x}, \ldots\right)$ such that

$$
\mathscr{P}_{\tau_{k}}\left(\varphi, \varphi_{x}, \ldots\right) \equiv \partial_{x} \mathscr{Q}_{(k)}\left(\varphi, \varphi_{x}, \ldots\right)
$$


or, as was pointed by Ferapontov [9], this means that the integral $I$ is the conservation law for any of the systems (2.8).

From Theorem 2.1 we have now that the flows (2.8) commute with all the local Hamiltonian fluxes generated by the local functionals (2.2) since they conserve both the Hamiltonian structure and the corresponding Hamiltonian functions.

3. The Whitham method and the "regularity" conditions. We come to Whitham's averaging procedure (see $[4,5,6,7,20,32]$ ). Recall that in the $m$-phase Whitham's method for the systems (2.7), we make a rescaling transformation $X=\epsilon \mathcal{X}, T=\epsilon t$ to obtain the system

$$
\epsilon \varphi_{T}^{i}=Q^{i}\left(\varphi, \epsilon \varphi_{X}, \epsilon^{2} \varphi_{X X}, \ldots\right),
$$

and then try to find the functions

$$
S(X, T)=\left(S^{1}(X, T), \ldots, S^{m}(X, T)\right),
$$

and $2 \pi$-periodic with respect to each $\theta^{\alpha}\left(\theta=\left(\theta^{1}, \ldots, \theta^{m}\right)\right)$ functions

$$
\Phi^{i}(\theta, X, T, \epsilon)=\sum_{k=0}^{\infty} \epsilon^{k} \Phi_{(k)}^{i}(\theta, X, T),
$$

such that the functions

$$
\varphi^{i}(\theta, X, T, \epsilon)=\sum_{k=0}^{\infty} \epsilon^{k} \Phi_{(k)}^{i}\left(\theta+\frac{S(X, T)}{\epsilon}, X, T\right)
$$

satisfy system (3.1) at any $\theta$ in any order of $\epsilon$.

It follows that $\Phi_{(0)}^{i}(\theta, X, T)$ at any $X$ and $T$ gives the exact $m$-phase solution of (2.7) depending on some parameters $U=\left(U^{1}, \ldots, U^{N}\right)$ and initial phases $\theta_{0}=\left(\theta_{0}^{1}, \ldots, \theta_{0}^{m}\right)$ and, besides that

$$
S_{T}^{\alpha}=\omega^{\alpha}(U(X, T)), \quad S_{X}^{\alpha}=k^{\alpha}(U(X, T)),
$$

where $\omega^{\alpha}(U)$ and $k^{\alpha}(U)$ are, respectively, the frequencies and the wave numbers of the corresponding $m$-phase solution of (2.7).

The conditions of the compatibility of system (3.1) in the first order of $\epsilon$ together with

$$
k_{T}^{\alpha}=\omega_{X}^{\alpha}
$$

give us Whitham's system of equations on the parameters $U(X, T)$,

$$
U_{T}^{v}=V_{\mu}^{v}(U) U_{X}^{\mu},
$$

which is the quasi-linear system of hydrodynamic type.

The first procedure of averaging the local field-theoretical Poisson brackets was proposed in $[5,6,7]$ by Dubrovin and Novikov. This procedure permits to obtain the local Poisson brackets of hydrodynamic type

$$
\left\{U^{v}(X), U^{\mu}(Y)\right\}=g^{v \mu}(U) \delta^{\prime}(X-Y)+b_{\gamma}^{v \mu}(U) U_{X}^{\gamma} \delta(X-Y),
$$


for Whitham's system (3.7) from the local Hamiltonian structure

$$
\left\{\varphi^{i}(x), \varphi^{j}(y)\right\}=\sum_{k \geq 0} B_{k}^{i j}\left(\varphi, \varphi_{x}, \ldots\right) \delta^{(k)}(x-y),
$$

for the initial system (2.7).

The method of Dubrovin and Novikov is based on the presence of $N$ (equal to the number of parameters $U^{v}$ of the family of $m$-phase solutions of (2.7)) local integrals

$$
I^{v}=\int \mathscr{P}^{v}\left(\varphi, \varphi_{x}, \ldots\right) d x
$$

commuting with the Hamiltonian function (2.2) and with each other

$$
\left\{I^{v}, H\right\}=0, \quad\left\{I^{v}, I^{\mu}\right\}=0,
$$

and can be written in the following form.

We calculate the pairwise Poisson brackets of the densities $\mathscr{P}^{v}$ in the form

$$
\left\{\mathscr{P}^{v}(x), \mathscr{P}^{\mu}(y)\right\}=\sum_{k \geq 0} A_{k}^{v \mu}\left(\varphi, \varphi_{x}, \ldots\right) \delta^{(k)}(x-y),
$$

where

$$
A_{0}^{v \mu}\left(\varphi, \varphi_{x}, \ldots\right) \equiv \partial_{x} Q^{v \mu}\left(\varphi, \varphi_{x}, \ldots\right)
$$

according to (3.11). Then the Dubrovin-Novikov bracket on the space of functions $U(X)$ can be written in the form

$$
\left\{U^{v}(X), U^{\mu}(Y)\right\}=\left\langle A_{1}^{v \mu}\right\rangle(U) \delta^{\prime}(X-Y)+\frac{\partial\left\langle Q^{v \mu}\right\rangle}{\partial U^{\gamma}} U_{X}^{\gamma} \delta(X-Y),
$$

where $\langle\cdot\rangle$ means the averaging on the family of $m$-phase solutions of (2.7) given by the formula, strictly speaking, this formula is valid for the generic set of the wave numbers $k^{\alpha}$, but we should use, in any case, the second part of it for the averaged quantities to obtain the right procedure (here $k^{\alpha}$ are continuous parameters on the family of the $m$-phase solutions),

$$
\begin{aligned}
\langle F\rangle & =\lim _{c \rightarrow \infty} \frac{1}{2 c} \int_{-c}^{c} F\left(\varphi, \varphi_{x}, \ldots\right) d x \\
& =\frac{1}{(2 \pi)^{m}} \int_{0}^{2 \pi} \cdots \int_{0}^{2 \pi} F\left(\Phi, k^{\alpha}(U) \Phi_{\theta^{\alpha}}, \ldots\right) d^{m} \theta,
\end{aligned}
$$

and we choose the parameters $U^{v}$ such that they coincide with the values of $I^{v}$ on the corresponding solutions

$$
U^{v}=\left\langle P^{v}(x)\right\rangle
$$

The Jacobi identity for the averaged bracket (3.14) in the general case was proved in [23] (for the systems having also local Lagrangian formalism, there was a proof in [25]). 
Note also that we get here the Poisson bracket only if we average the initial Hamiltonian structure on the full family of $m$-phase solutions (see [23, 28]). More precise requirements will be formulated when describing the averaging procedure in the nonlocal case.

Brackets (3.8) can be described from the differential-geometrical point of view. Namely, for the nondegenerated tensor $g^{\nu \mu}$, we have that it should be a flat contravariant metric and the values

$$
\Gamma_{\mu \gamma}^{\nu}=-g_{\mu \lambda} b_{\gamma}^{\lambda \nu}
$$

should be the Levi-Civita connection for the metric $g_{v \mu}$ (with lower indices). The brackets (3.8) with the degenerated tensor are more complicated but also have a nice geometrical structure (see [18]).

The nonlocal Poisson brackets (2.6) are the generalization of local Poisson brackets of Dubrovin and Novikov and are closely connected with the integrability of systems of hydrodynamic type reducible to the diagonal form [31]. Namely, the system reducible to the diagonal form and Hamiltonian, with respect to the bracket (2.6), satisfies (see $[9,10,11,16])$ the so-called "semi-Hamiltonian" property introduced by Tsarëv [31] and can be integrated by Tsarëv's "generalized hodograph method." In [3], the investigation of the equivalence of "semi-Hamiltonian" properties, introduced by Tsarëv, and the Hamiltonian properties with respect to the bracket (2.6) can also be found.

We also point here that the questions of integrability of Hamiltonian with respect to (2.6) systems, which cannot be written in the diagonal form, were studied in [12, $13,14,15]$.

The procedure of averaging of the nonlocal Poisson brackets in Whitham's method and the proof of the Jacobi identity for the averaged nonlocal bracket resemble those for the local brackets. However, the difference in formulas of averaging and in the proof contain very many essential things and also many considerations valuable for the local case that cannot be used in the nonlocal one. So, we should make here the consideration for the nonlocal case.

The $m$-phase solutions of (2.7)

$$
\varphi^{i}(x, t)=\Phi^{i}\left(\omega t+k x+\theta_{0}\right)
$$

where

$$
\omega=\left(\omega^{1}, \ldots, \omega^{m}\right), \quad k=\left(k^{1}, \ldots, k^{m}\right),
$$

are the $2 \pi$-periodic solutions of the system

$$
\omega^{\alpha} \Phi_{\theta^{\alpha}}^{i}-Q^{i}\left(\Phi, k^{\alpha} \Phi_{\theta^{\alpha}}, k^{\alpha} k^{\beta} \Phi_{\theta^{\alpha} \theta^{\beta}}, \ldots\right)=0, \quad \alpha=1, \ldots, m,
$$

depending on the parameters $\omega$ and $k$. So we assume that we have, from (3.20) for the generic $\omega$ and $k$, the finite-dimensional subspace $\mu_{\omega, k}$ (in the space of $2 \pi$-periodic with respect to each $\theta^{\alpha}$ functions) parameterized by the initial phase shifts $\theta_{0}^{\alpha}$ and 
may be also by some additional parameters $r^{1}, \ldots, r^{h}$. For the multiphase case $(m \geq 2)$, it is essential that the closure of any orbit generated by the vectors $\left(\omega_{1}, \ldots, \omega_{m}\right)$ and $\left(k_{1}, \ldots, k_{m}\right)$ in the $\theta$-space is the full $m$-dimensional torus $\mathbf{T}^{m}$. For the case of "rationally-dependent" $\omega_{1}, \ldots, \omega_{m}$ and $k_{1}, \ldots, k_{m}$ and $m \geq 3$, we have that the operators (3.20) are independent of each of such closed submanifolds in $\mathbf{T}^{m}$ which can have dimensionality less than $m$. The functions from $\mu_{\omega, k}$ can be found in this case from the additional requirement that they are the $m$-phase solutions for (2.8) (with some $\left.\Omega_{(k)}^{\alpha}(\omega, k, r)\right)$ and the systems generated by the functionals $I^{v}$ (see (3.22) later) with some $\omega^{\alpha v}(\omega, k, r)$. All these requirements uniquely define the finite-dimensional spaces $\mu_{\omega, k}$ which continuously depend on the parameters $\omega$ and $k$.

Combining all such $\mu_{\omega, k}$ at different $\omega$ and $k$, we obtain that the $m$-phase solutions of the system (2.7) can be parameterized by $N=2 m+h$ parameters $\left(U^{1}, \ldots, U^{N}\right)$ invariant with respect to the initial shifts of $\theta^{\alpha}$ and the initial phase shifts $\theta_{0}^{\alpha}$ after the choice of some "initial" functions $\Phi_{(i n)}^{i}(\theta, U)$ corresponding to the zero initial phases. The joint of the submanifolds $\mu_{\omega, k}$ at all $\omega$ and $k$ gives us the submanifold $\mu$ in the space of $2 \pi$-periodic with respect to each $\theta^{\alpha}$ functions which corresponds to the full family of $m$-phase solutions of (2.7).

For Whitham's procedure, we should now require some "regularity" properties of the system of constraints (3.20) these properties are the following.

(I) We require that the linearized system (3.20) at any "point" of $\mu_{\omega, k}$ has exactly $h+m=N-m$ solutions ("right eigenvectors") $\xi_{(q) \omega, k}(\theta, r)$ for the generic $\omega$ and $k$ given by the vectors tangential to $\mu_{\omega, k}$ which are the derivatives $\Phi_{\theta^{\alpha}}(\theta, r, \omega, k)$ and $\Phi_{r^{q}}(\theta, r, \omega, k)$ (at the fixed values of $\omega$ and $k$ ).

(II) We also require that the number of the linearly independent "left eigenvectors" $\kappa_{(q)} \omega, k(\theta, r)$ orthogonal to the image of the introduced linear operator is exactly the same $(N-m)$ as the number of the "right eigenvectors" $\xi_{(q) \omega, k}(\theta, r)$ for the generic $\omega$ and $k$. In addition, we assume that $\kappa_{(q)} \omega, k(\theta, r)$ also depend continuously on the parameters $U^{v}$ on $M$.

The requirements (I) and (II) are closely connected with Whitham's procedure and the asymptotic solutions (3.4). Indeed, it is not very difficult to see that every $k$ th term in the expansion (3.4) is determined by the above defined linear system with the nontrivial right-hand part depending on the previous terms of (3.4). We suppose that this system is resolvable on the space of $2 \pi$-periodic with respect to each $\theta^{\alpha}$ functions if the right-hand part is orthogonal to all the "right eigenvectors" $\kappa_{(q) \omega, k}(\theta, r)$ for the corresponding ( $\omega=S_{T}, k=S_{X}, r$ ). If so, the solution of this system can be found modulo the "left eigenvectors" $\xi_{(q) \omega, k}(\theta, r)$ with the same $\omega, k$, and $r$ defined by the zero term of (3.4). So we can find in the generic situation the unique $\Phi_{(k)}(\theta, X, T)$ for $k \geq 1$ from the $N-m$ compatibility conditions of the same system in the order $k+1$ when the compatibility conditions in the first order of $\epsilon$, together with

$$
k_{T}=\omega_{X},
$$

give us Whitham's system of (3.7). We assume that $\Phi_{(k)}(\theta, X, T)$ depend continuously on $X$ and $T$ and so, are also well defined for the nongeneric $k_{1}, \ldots, k_{m}$ and $\omega_{1}, \ldots, \omega_{m}$ in the multiphase Whitham's method. 
We now discuss the requirements (I) and (II) from the Hamiltonian point of view.

First of all for the procedure of averaging of the bracket (1.1), we need the set of the integrals $I^{v}, v=1, \ldots, N$, like in the procedure of Dubrovin and Novikov, that is, satisfying the following requirements.

(A) Every $I^{v}$ is the local functional $I^{v}=\int \mathscr{P}^{v}\left(\varphi, \varphi_{x}, \ldots\right) d x$, which generates the local flow

$$
\varphi_{t^{v}}^{i}=Q_{(v)}^{i}\left(\varphi, \varphi_{x}, \ldots\right)
$$

with respect to the bracket (1.1).

As was pointed above, we should have for this that the local flows (2.8) defined from the bracket (1.1) in the "canonical" (or "irreducible") form conserve all the $I^{v}$, that is, the time derivatives of the corresponding $\mathscr{P}^{v}\left(\varphi, \varphi_{x}, \ldots\right)$ with respect to each of the flows (2.8) are the total derivatives with respect to $x$

$$
\frac{d}{d t^{k}} \mathscr{P}^{v}\left(\varphi, \varphi_{x}, \ldots\right) \equiv \partial_{x} F_{(k)}^{v}\left(\varphi, \varphi_{x}, \ldots\right)
$$

for some functions $F_{(k)}^{v}\left(\varphi, \varphi_{x}, \ldots\right)$.

(B) All $I^{v}$ commute with each other and with the Hamiltonian function (2.2), that is, $\left\{I^{v}, I^{\mu}\right\}=0,\left\{I^{v}, H\right\}=0$.

(C) The averaged densities $\left\langle\mathscr{P}^{v}\right\rangle$

$$
\begin{aligned}
\left\langle\mathscr{P}^{v}\right\rangle & =\lim _{c \rightarrow \infty} \frac{1}{2 c} \int_{-c}^{c} \mathscr{P}^{v}\left(\varphi, \varphi_{x}, \ldots\right) d x \\
& =\frac{1}{(2 \pi)^{m}} \int_{0}^{2 \pi} \cdots \int_{0}^{2 \pi} \mathscr{P}^{v}\left(\Phi, k^{\alpha} \Phi_{\theta^{\alpha}}, \ldots\right) d^{m} \theta
\end{aligned}
$$

can be regarded as the independent coordinates $\left(U^{1}, \ldots, U^{N}\right)$ on the family of $m$-phase solutions of (2.7). Here again we should use everywhere the second part of formula (3.24) for the averaged values on $M$ for the right procedure as will be shown later.

From the requirements above, we immediately obtain that the flows (3.22) commute with our initial system (2.7) and with each other.

From Theorem 2.1, we obtain also that the commutative flows (2.8) defined by the Poisson bracket also commute with (2.7) and (3.22) since they conserve the corresponding Hamiltonian functions and the Hamiltonian structure (2.1).

We can consider the functionals

$$
\begin{aligned}
\bar{I}^{v} & =\lim _{c \rightarrow \infty} \frac{1}{2 c} \int_{-c}^{c} \mathscr{P}^{v}\left(\varphi, \varphi_{x}, \ldots\right) d x, \\
\bar{H} & =\lim _{c \rightarrow \infty} \frac{1}{2 c} \int_{-c}^{c} \mathscr{P}_{H}\left(\varphi, \varphi_{x}, \ldots\right) d x,
\end{aligned}
$$

on the space of the quasiperiodic functions (with $m$ quasiperiods). 
It is easy to see now that the local fluxes (2.7), (2.8), and (3.22) being considered on the space of the quasiperiodic functions also conserve the values of $\bar{I}^{v}$ and $\bar{H}$ and commute with each other since these properties can be expressed just as the local relations containing $\varphi, \varphi_{x}, \ldots$ and the time derivatives of the densities $\mathscr{P}^{v}\left(\varphi, \varphi_{x}, \ldots\right)$, $\mathscr{P}_{H}\left(\varphi, \varphi_{x}, \ldots\right)$ at the same point $x$.

Now we can conclude that all the fluxes (2.8) and (3.22) leave the family of $m$-phase solutions given by (3.20) invariant and can generate on it only the linear shifts of the initial phases $\theta_{0}^{\alpha}$ which follow from the commutativity of the flows

$$
\begin{aligned}
& \varphi_{T^{k}}^{i}(\theta)=S_{(k)}^{i}\left(\varphi, k^{\alpha} \varphi_{\theta^{\alpha}}, k^{\alpha} k^{\beta} \varphi_{\theta^{\alpha} \theta^{\beta}}, \ldots\right), \\
& \varphi_{t^{v}}^{i}(\theta)=Q_{(v)}^{i}\left(\varphi, k^{\alpha} \varphi_{\theta^{\alpha}}, k^{\alpha} k^{\beta} \varphi_{\theta^{\alpha} \theta^{\beta}}, \ldots\right),
\end{aligned}
$$

with the flows $\varphi_{t^{\alpha}}^{i}=\varphi_{\theta^{\alpha}}^{i}$ and

$$
\varphi_{t}^{i}=Q^{i}\left(\varphi, k^{\alpha} \varphi_{\theta^{\alpha}, k^{\alpha}} k^{\beta} \varphi_{\theta^{\alpha} \theta^{\beta}}, \ldots\right),
$$

on the space of $2 \pi$-periodic with respect to each $\theta^{\alpha}$ functions and the conservation of the functionals $\bar{I}^{v}$ (i.e., $U^{v}$ on $M$ ) by the flows (3.27). (Here $k^{\alpha}$ are $m$ quasiperiods of the function $\varphi(x)$.) So we obtain that our family of $m$-phase solutions of (2.7) is also the family of $m$-phase solutions for (2.8) and (3.22) and we assume also the existence of the solutions (3.4) for these systems based on the family $\mathcal{M}$.

We can also conclude that in our situation the variational derivatives of the functionals (3.25) and (3.26), with respect to $\varphi(\theta)$ at the points of the submanifold $\mathcal{M}$, are the linear combinations of the corresponding "right eigenvectors" $\kappa_{(q)}\left(\theta+\theta_{0}, U\right)$ (see $[4,6,7]$ and the references therein). Indeed from the conservation of the functionals (3.25) and (3.26) by the flows $\varphi_{t^{\alpha}}^{i}=\varphi_{\theta^{\alpha}}^{i}$ and

$$
\varphi_{t}^{i}=Q^{i}\left(\varphi, k^{\alpha} \varphi_{\theta^{\alpha}}, \ldots\right),
$$

we can conclude that the convolution of their derivatives with respect to $\varphi^{i}(\theta)$ with the system of constraints (3.20) is identically zero for all the periodic functions with respect to all $\theta^{\alpha}$ and for any $\left(k^{1}, \ldots, k^{m}\right)$ and $\left(\omega^{1}, \ldots, \omega^{m}\right)$. So we can take the variational derivative of the corresponding expression with respect to $\varphi^{j}\left(\theta^{\prime}\right)$ and then omit the second variational derivative of $\bar{I}^{v}$ and $\bar{H}$ according to the conditions (3.20). After that we obtain that the variational derivatives of $\bar{I}^{v}$ and $\bar{H}$ are also orthogonal to the image of the linearized operator (3.20) at the points of $\mathcal{M}$ and so are the linear combinations of $\kappa_{(q)}\left(\theta+\theta_{0}, U\right)$ on $\mu$.

LEMMA 3.1. Suppose the properties (A), (B), (C) and (I), (II) are satisfied for our family of $m$-phase solutions of (2.7). We put

$$
U^{v}=\left\langle\mathscr{P}^{v}\right\rangle=\bar{I}^{v}
$$

on the space $M$, and then define the functions $k^{\alpha}=k^{\alpha}(U)$ on the submanifold $M$.

Then the functionals $K^{\alpha}=k^{\alpha}(\bar{I}[\varphi])$ on the space of $2 \pi$-periodic with respect to each $\theta^{\alpha}$ functions (and also at the space of quasiperiodic functions $\varphi(x)$ with $m$ quasiperiods) have the zero variational derivatives on the submanifold $M$. 
Proof. As we have from (II), the maximal number of the linearly independent variational derivatives of $\bar{I}^{v}$ on $M$ is $h+m=N-m$. So we have $m$ linearly independent relations

$$
\sum_{v=1}^{N} \lambda_{v}^{\alpha}(U) \frac{\delta \bar{I}^{v}}{\delta \varphi(\theta)} \equiv 0, \quad \alpha=1, \ldots, m
$$

(where $\varphi(\theta)=\left(\varphi^{1}(\theta), \ldots, \varphi^{m}(\theta)\right)$ ) being considered at the given $k^{1}, \ldots, k^{m}$ at any point of $\mathcal{M}$, (or in other words

$$
\sum_{v=1}^{N} \lambda_{v}^{\alpha}(U) \frac{\delta \bar{I}^{v}}{\delta \varphi(x)} \equiv 0, \quad \alpha=1, \ldots, m
$$

when considered in the space of functions with $m$ quasiperiods). We have here the standard expression for the variational derivative and the formula (3.24) for $\bar{I}^{v}$.

Since we can obtain the change of these linear combinations of the functionals $\bar{I}^{v}$ on $\mathcal{M}$ only due to the variations of $k$ in (3.24) but not of $\varphi^{i}(\theta)$ (or in other words only if we have the nonbounded variations of $\varphi^{i}(x)$ after the variations of the quasiperiods) we have on $\mu$

$$
\sum_{v=1}^{N} \lambda_{v}^{\alpha}(U) d U^{v}=\sum_{\beta=1}^{m} \mu_{\beta}^{(\alpha)}(U) d k^{\beta}(U)
$$

for some functions $\mu_{\beta}^{(\alpha)}(U)$.

If $U^{v}$ are the coordinates on $\mu$ then the matrix $\mu_{\beta}^{(\alpha)}$ has the full rank and is reversible. So we obtain the differentials $d k^{\beta}$ as the linear combinations of differentials $\sum_{v=1}^{N} \lambda_{v}^{\alpha}(U) d U^{v}$ corresponding to the functionals with zero derivatives on $\mu$

$$
d k^{\beta}=\sum_{\alpha=1}^{m}\left(\mu^{-1}\right)_{(\alpha)}^{\beta} \sum_{v=1}^{N} \lambda_{\nu}^{(\alpha)}(U) d U^{v} .
$$

So Lemma 3.1 now follows from (3.31).

REMARK 3.2. As can be seen from the proof of Lemma 3.1, the variational derivatives of $\bar{I}^{v}$ on $M$ should span all the $(N-m)$-dimensional linear space generated by all $\kappa_{(q)}\left(\theta+\theta_{0}, U\right)$ if we want to take $\left\langle\mathscr{P}^{v}\right\rangle$ as the independent coordinates on $\mathcal{M}$. It is essential also that we consider the full family of $m$-phase solutions given by (3.20) at different $\omega$ and $k$, but not its "subspace," and have $m$ independent relations (3.33) on $N$ differentials $d U^{v}$ from $m$ relations (3.31).

REMARK 3.3. We note that (3.32) was introduced at first by Novikov in [27] as the definition of the $m$-phase solutions for the KdV equation.

We now prove a technical lemma which we will need later.

LEMMA 3.4. Introduce the additional densities

$$
\prod_{i(k)}^{v}\left(\varphi, \varphi_{x}, \ldots\right) \equiv \frac{\partial \mathscr{P}^{v}\left(\varphi, \varphi_{x}, \ldots\right)}{\partial \varphi_{k x}^{i}}
$$

for $k \geq 0$, where $\varphi_{k x}^{i} \equiv \partial^{k} / \partial x^{k} \varphi^{i}(x)$. 
Then on the submanifold $M$ the relation

$$
\begin{aligned}
\sum_{v=1}^{N} \frac{\partial k^{\alpha}}{\partial U^{v}} \frac{1}{(2 \pi)^{m}} \int_{0}^{2 \pi} \cdots \int_{0}^{2 \pi} \sum_{p \geq 1} p k^{\beta_{1}}(U) \cdots k^{\beta_{p-1}}(U) \Phi_{(i n) \theta^{\beta} \theta^{\beta_{1}} \cdots \theta^{\beta} p-1}^{i}(\theta, U) \\
\times \prod_{i(p)}^{v}\left(\Phi_{(i n)}(\theta, U), k^{\gamma} \Phi_{(i n) \theta \gamma}(\theta, U), \ldots\right) d^{m} \theta \equiv \delta_{\beta}^{\alpha}
\end{aligned}
$$

holds at any $U$ and $\theta_{0}$.

PROoF. According to Lemma 3.1 we should not take into account the variations of the form of $\Phi_{(i n)}\left(\theta+\theta_{0}, U\right)$ when we consider the change of the functionals $k^{\alpha}(\bar{I})$ on $\mu$. So the only source for the change of these functionals on $M$ is the dependence on the wave numbers $k$ in the expressions

$$
\bar{I}^{v}=\frac{1}{(2 \pi)^{m}} \int_{0}^{2 \pi} \cdots \int_{0}^{2 \pi} \mathscr{P}^{v}\left(\Phi_{(i n)}, k^{\gamma} \Phi_{(i n) \theta^{\gamma}}, k^{\gamma} k^{\delta} \Phi_{(i n) \theta^{\gamma} \theta^{\delta}}, \ldots\right) d^{m} \theta .
$$

So we can write

$$
d\left(\left.k^{\alpha}(\bar{I})\right|_{\mu}\right)=\left.\sum_{v=1}^{N} \frac{\partial k^{\alpha}}{\partial U^{v}}(U) \frac{\partial \bar{I}^{v}[\varphi]}{\partial k^{\beta}}\right|_{\mu} d k^{\beta},
$$

where the values of $\partial \bar{I}^{v}[\varphi] / \partial k^{\beta}$ on $M$ are given by the integral expressions from (3.36). Since the values of the functionals $k^{\alpha}(\bar{I})$ on $\mu$ coincide by the definition with the wave numbers $k^{\alpha}$ we obtain the relation (3.36). Lemma 3.4 is proved.

For the evolution of the densities $\mathscr{P}^{v}\left(\varphi, \varphi_{x}, \ldots\right)$ according to our system (2.7) we can also write the relations

$$
\frac{d}{d t} \mathscr{P}^{v}\left(\varphi, \varphi_{x}, \ldots\right) \equiv \partial_{x} Q^{v H}\left(\varphi, \varphi_{x}, \ldots\right),
$$

and Whitham's system (3.7) can also be written in the following "conservative" form

$$
\partial_{T} U^{v}=\partial_{X}\left\langle Q^{v H}\right\rangle, \quad v=1, \ldots, N,
$$

for the parameters $U^{v}$.

From the existence of the series (3.4) on the space of $2 \pi$-periodic with respect to $\theta$ functions, it can be shown that this form gives us the system equivalent to (3.7) in the generic situation, and all the other local conservation laws of the form (3.39) (if they exist) give us the equation

$$
\partial_{T}\langle\mathscr{P}\rangle=\partial_{X}\left\langle Q^{H}\right\rangle
$$

compatible with (3.40) for the full set of parameters $U^{v}$ (see [4, 5, 6, 7, 20, 32]).

The conservative form (3.40) of Whitham's system will be very convenient in our considerations on the averaging of Hamiltonian structures.

Now we make some "regularity" requirements about the joint $\mu$ of the submanifolds $\mu_{\omega, k}$ at all $\omega$ and $k$ corresponding to the $m$-phase quasiperiodic solutions of the system (2.7), that is, 
(III) we require that $\mathcal{M}$ is an $(N+m)$-dimensional submanifold in the space of the $2 \pi$-periodic with respect to each $\theta^{\alpha}$ functions.

The property (III) means nothing but the fact that the shapes of the solutions of (3.20) are all different at different $\omega$ and $k$ in the space of the $2 \pi$-periodic vectorfunctions of $\theta$ so that $\omega$ and $k$ can be reconstructed from them. It is easy to see that this requirement corresponds to the generic situation. We will use the property (III) for our procedure of averaging of bracket (2.1).

We will work with the full family of $2 \pi$-periodic solutions of (3.20) the functions of which will also depend on the "slow" variables $X$ and $T$. To define this submanifold in the space of functions $\varphi(\theta, X, T)$ with the system of constraints like (3.20), we should extend the coordinates $U^{v}$ as the functionals of $\varphi(\theta)$ in the vicinity of our submanifold $M$. This can be easily done (see [23]) by the following way.

Introduce $N$ different functionals

$$
A^{v}=\frac{1}{(2 \pi)^{m}} \int_{0}^{2 \pi} \cdots \int_{0}^{2 \pi} a^{v}\left(\varphi, \varphi_{\theta^{\alpha}}, \varphi_{\theta^{\alpha} \theta^{\beta}}, \ldots\right) d^{m} \theta
$$

such that their values $\bar{A}^{v}$ are functionally independent on the functions, from the submanifold $M$. Then we can express $U^{v}=U^{v}(\bar{A})$ in terms of $\bar{A}^{v}$ on $M$ and after that extend them as the functionals $U^{v}(A)$ on the space of $2 \pi$-periodic with respect to each $\theta^{\alpha}$ functions.

We also expand the coordinates $\theta_{0}^{\alpha}$ (see [23]) in the vicinity of $\mu$ by introduction of, say, functionals

$$
B^{\alpha}[\varphi(\theta)]=\frac{1}{(2 \pi)^{m}} \int_{0}^{2 \pi} \cdots \int_{0}^{2 \pi} \varphi_{\theta^{\alpha}}(\theta) \Phi_{(i n)}(\theta, U[\varphi]) d^{m} \theta
$$

which are zero if $\varphi(\theta)=\Phi_{(i n)}(\theta, U[\varphi])$. In the generic situation, we can locally express the values of $\theta_{0}^{\alpha}$ on $\mu$ in terms of $\bar{B}^{\alpha}$ and after that put $\theta_{0}^{\alpha}=\theta_{0}^{\alpha}(B[\varphi])$ in the corresponding local coordinate maps in the vicinity of $\mu$.

We consider the system

$$
\varphi^{i}(\theta, X)-\Phi_{(i n)}^{i}\left(\theta+\theta_{0}[\varphi], U[\varphi]\right) \equiv 0,
$$

where $\theta_{0}^{\alpha}[\varphi]$ and $U^{v}[\varphi]$ are the functionals in the vicinity of $\mu$ as the system of constraints which defines $\mathcal{M}$ in the space of $2 \pi$-periodic with respect to each $\theta^{\alpha}$ functions.

We can see now that the linearized system (3.44)

$$
\frac{1}{(2 \pi)^{m}} \int_{0}^{2 \pi} \cdots \int_{0}^{2 \pi}\left(L_{j\left[U, \theta_{0}\right]}^{i}\left(\theta, \theta^{\prime}\right) \delta \Phi^{j}\left(\theta^{\prime}\right)\right) d^{m} \theta^{\prime}=0
$$

where

$$
\begin{aligned}
L_{j\left[U, \theta_{0}\right]}^{i}\left(\theta, \theta^{\prime}\right) \equiv & \delta_{j}^{i} \delta\left(\theta-\theta^{\prime}\right)-\sum_{\alpha=1}^{m} \Phi_{(i n) \theta^{\alpha}}^{i}\left(\theta+\theta_{0}[\varphi], U[\varphi]\right) \times \frac{\delta \theta_{0}^{\alpha}[\varphi]}{\delta \varphi^{j}\left(\theta^{\prime}\right)} \\
& -\sum_{v=1}^{N} \Phi_{(i n) U^{v}}^{i}\left(\theta+\theta_{0}[\varphi], U[\varphi]\right) \times \frac{\delta U_{0}^{v}[\varphi]}{\delta \varphi^{j}\left(\theta^{\prime}\right)}
\end{aligned}
$$


at any point $\left(U, \theta_{0}\right)$ of $\mathcal{M}$ has exactly $N+m$ solutions $\tilde{\xi}_{(p)\left[U, \theta_{0}\right]}(\theta)$ corresponding to the tangential to $\mathcal{M}$ vectors $\Phi_{(i n) \theta^{\alpha}}$ and $\Phi_{(i n) U^{v}}, \alpha=1, \ldots, m, v=1, \ldots, N$.

It is evident also that all the "left eigenvectors" $\tilde{\kappa}_{(p)\left[U, \theta_{0}\right]}(\theta)$ orthogonal to the image of $\hat{L}$ are given by the variational derivatives $\delta \theta_{0}^{\alpha}[\varphi] / \delta \varphi^{j}(\theta)$ and $\delta U_{0}^{v}[\varphi] / \delta \varphi^{j}(\theta)$.

From the invariance of the submanifold $\mu$ with respect to the flows (2.8) and (3.22), we can also write the relations

$$
\begin{aligned}
& \frac{1}{(2 \pi)^{m}} \int_{0}^{2 \pi} \cdots \int_{0}^{2 \pi} L_{j\left[U, \theta_{0}\right]}^{i}\left(\theta, \theta^{\prime}\right) \\
& \quad \times S_{(k)}^{j}\left(\Phi_{(i n)}\left(\theta^{\prime}+\theta_{0}, U\right), k^{\alpha} \Phi_{(i n) \theta^{\alpha}}\left(\theta^{\prime}+\theta_{0}, U\right), \ldots\right) d^{m} \theta^{\prime} \equiv 0, \\
& \frac{1}{(2 \pi)^{m}} \int_{0}^{2 \pi} \cdots \int_{0}^{2 \pi} L_{j\left[U, \theta_{0}\right]}^{i}\left(\theta, \theta^{\prime}\right) \\
& \quad \times Q_{(v)}^{j}\left(\Phi_{(i n)}\left(\theta^{\prime}+\theta_{0}, U\right), k^{\alpha} \Phi_{(i n) \theta^{\alpha}}\left(\theta^{\prime}+\theta_{0}, U\right), \ldots\right) d^{m} \theta^{\prime} \equiv 0,
\end{aligned}
$$

for any $i, k$ at any point $\left(U, \theta_{0}\right)$ of $\mathcal{M}$ where $k^{\alpha}=k^{\alpha}[\Phi]$ can be considered now as the values of the corresponding functionals on $\mu$.

We introduce the space of functions $\varphi(\theta, X, T)$ depending on "slow" parameters $X$ and $T$, and $2 \pi$-periodic with respect to each $\theta^{\alpha}$. Systems (3.44) being considered independently at different $X$, give us the system of constraints defining the subspace $\mathcal{M}^{\prime}$ in the space of functions $\varphi(\theta, X)$ corresponding to $m$-phase solutions of (2.7) depending also on the parameters $X$ and $T$.

After the introduction of the "modified" constraints (3.44)

$$
\begin{aligned}
G_{\left[U, \theta_{0}\right]}^{i}(\theta, X)=\frac{1}{(2 \pi)^{m}} \int_{0}^{2 \pi} \cdots \int_{0}^{2 \pi} & L_{j\left[U, \theta_{0}\right]}^{i}\left(\theta, \theta^{\prime}\right) \\
& \times\left(\varphi^{j}\left(\theta^{\prime}\right)-\Phi_{(i n)}^{j}\left(\theta^{\prime}+\theta_{0}[\varphi], U[\varphi]\right)\right) d^{m} \theta^{\prime},
\end{aligned}
$$

we can take $U^{v}(X), \theta_{0}^{\alpha}(X)$, and $G_{\left[U, \theta_{0}\right]}^{i}(\theta, X)$ such that

$$
\begin{aligned}
\frac{1}{(2 \pi)^{m}} \int_{0}^{2 \pi} \cdots \int_{0}^{2 \pi} & \tilde{\kappa}_{(p)[U]}\left(\theta+\theta_{0}(X)\right) \\
& \times G_{\left[U, \theta_{0}\right]}^{i}(\theta, X) d^{m} \theta \equiv 0, \quad p=1, \ldots, N+m,
\end{aligned}
$$

as the coordinates are in the vicinity of $\mathcal{M}^{\prime}$ instead of the $\varphi^{i}(\theta, X)$ since we can find, uniquely, $\varphi^{i}(\theta, X)$ from

$$
\frac{1}{(2 \pi)^{m}} \int_{0}^{2 \pi} \cdots \int_{0}^{2 \pi} L_{j\left[U, \theta_{0}\right]}^{i}\left(\theta, \theta^{\prime}\right)\left(\varphi^{j}\left(\theta^{\prime}\right)-\Phi_{(i n)}^{j}\left(\theta^{\prime}+\theta_{0}, U\right)\right) d^{m} \theta^{\prime}=G_{\left[U, \theta_{0}\right]}^{i}(\theta, X)
$$

if we have conditions (3.50) and the values of $U^{v}(X)$ and $\theta_{0}^{\alpha}(X)$. This system of constraints is different from the system introduced in [23].

REMARK 3.5. Certainly, we have some freedom in the choice of the constraints $G^{i}(\theta, X)$. For example, we can also take the expressions (3.44) as the system of constraints defining $\mathcal{M}^{\prime}$. We prefer to take the constraints in the form (3.49) just to fix the uniform orthogonality conditions (3.50) in the vicinity of $\mathcal{M}^{\prime}$. 
We also need another coordinate system in the vicinity of $\mathcal{M}^{\prime}$, which differs from the described above, by the transformation depending on the small parameter $\epsilon$ and singular at $\epsilon \rightarrow 0$. Namely, we recall our integrals (3.10)

$$
I^{v}=\int \mathscr{P}^{v}\left(\varphi, \varphi_{x}, \ldots\right) d x
$$

make a transformation $X=\epsilon \mathcal{X}$, and define the functionals

$$
J^{v}(X)=\frac{1}{(2 \pi)^{m}} \int_{0}^{2 \pi} \cdots \int_{0}^{2 \pi} \mathscr{P}^{v}\left(\varphi(\theta, X), \epsilon \varphi_{X}(\theta, X), \ldots\right) d^{m} \theta
$$

on the space of $2 \pi$-periodic with respect to each $\theta^{\alpha}$ functions $\varphi(\theta, X)$.

We also introduce the functionals

$$
\theta_{0}^{* \alpha}(X)=\theta_{0}^{\alpha}(X)-\theta_{0}^{\alpha}\left(X_{0}\right)-\frac{1}{\epsilon} \int_{X_{0}}^{X} k^{\alpha}\left(J\left(X^{\prime}\right)\right) d X^{\prime},
$$

for some fixed point $X_{0}$. We have identically

$$
\theta_{0}^{* \alpha}\left(X_{0}\right) \equiv 0
$$

As was shown in [23], we can also obtain the values of $U^{v}(X)$ and $\theta_{0}^{\alpha}(X)$ from $J^{v}(X)$, $\theta_{0}^{* \alpha}(X)$, and $\theta_{0}^{\alpha}\left(X_{0}\right)$ on $\mathcal{M}^{\prime}$ as the formal series in powers of $\epsilon$ and we have for these series

$$
\begin{aligned}
& U^{v}(X)\left[J, \theta_{0}^{*}\right]=J^{v}(X)+\sum_{k \geq 1} \epsilon^{k} u_{(k)}^{v}\left(J, J_{X}, \theta_{0 X}^{*}, \ldots\right), \\
& \theta_{0}^{\alpha}(X)\left[J, \theta_{0}^{*}\right]=\theta_{0}^{* \alpha}(X)+\theta_{0}^{\alpha}\left(X_{0}\right)+\frac{1}{\epsilon} \int_{X_{0}}^{X} k^{\alpha}\left(J\left(X^{\prime}\right)\right) d X^{\prime} .
\end{aligned}
$$

The form of the relation (3.56) will be important in our considerations, so we reproduce here the calculations from [23].

We recall that the values $J^{v}(X), \theta_{0}^{* \alpha}(X), \theta_{0}^{\alpha}\left(X_{0}\right)$, and $U^{\mu}(X)$ are connected on $\mathcal{M}^{\prime}$ by the relations (the definition of $J^{v}(X)$ )

$$
\begin{aligned}
& J^{v}(X)= \frac{1}{(2 \pi)^{m}} \int_{0}^{2 \pi} \cdots \int_{0}^{2 \pi} \mathscr{P}^{v}\left(\Phi_{(i n)}(\theta+s(X, \epsilon), U(X)),\right. \\
&\left.\epsilon \partial_{X} \Phi_{(i n)}(\theta+s(X, \epsilon), U(X)), \ldots\right) d^{m} \theta \\
&=\frac{1}{(2 \pi)^{m}} \int_{0}^{2 \pi} \cdots \int_{0}^{2 \pi} \mathscr{P}^{v}\left(\Phi_{(i n)}(\theta+s(X, \epsilon), U(X)),\right. \\
&\left.k^{\alpha}(J) \partial_{\theta^{\alpha}} \Phi_{(i n)}(\theta+s(X, \epsilon), U(X)), \ldots\right) d^{m} \theta \\
&+\sum_{k \geq 1} \epsilon^{k} \frac{1}{(2 \pi)^{m}} \int_{0}^{2 \pi} \cdots \int_{0}^{2 \pi} \mathscr{P}_{(k)}^{v}\left(\Phi_{(i n)}(\theta+s(X, \epsilon), U(X)), \ldots\right) d^{m} \theta,
\end{aligned}
$$

where

$$
s(X, \epsilon) \equiv \theta_{0}^{*}(X)+\theta_{0}\left(X_{0}\right)+\frac{1}{\epsilon} \int_{X_{0}}^{X} k\left(J\left(X^{\prime}\right)\right) d X^{\prime}
$$


and $\mathscr{P}_{(k)}^{v}\left(\Phi_{(i n)}(\theta+s(X, \epsilon), \ldots)\right)$ are local densities depending upon $\Phi_{(i n)}(\theta+s(X, \epsilon)$, $U(X))$ and their derivatives with respect to $U^{v}$ and $\theta^{\alpha}$ with the coefficients of types: $U_{X}(X), U_{X X}(X), \ldots, k(J), \partial_{X} k(J), \partial_{X}^{2} k(J), \ldots$, and $\theta_{0 X}^{*}(X), \theta_{0 X X}^{*}(X), \ldots$, are given by collecting together these terms, having the general multiplier $\epsilon^{k}$. The term corresponding to the zero power of $\epsilon$ is written separately.

After the integration with respect to $\theta$, which removes the singular at $\epsilon \rightarrow 0$ phase shift $\theta_{0}$ in the argument of $\Phi_{(i n)}$, we obtain on $\mathcal{M}^{\prime}$

$$
J^{v}(X)=\zeta^{v}(J, U)+\sum_{k \geq 1} \epsilon^{k} \zeta_{(k)}^{v}\left(U, U_{X}, \ldots, U_{k X}, J, J_{X}, \ldots, J_{k X}, \theta_{0 X}^{*}, \ldots, \theta_{0 k X}^{*}\right) .
$$

The sum in (3.60) contains the finite number of terms. The functions $\zeta_{(k)}^{v}$ and $\zeta^{v}$ are integrated with respect to $\theta$ functions $\mathscr{P}_{(k)}^{v}$, and $\mathscr{P}^{v}$, respectively.

So, since

$$
\zeta^{v}(J, U)=\frac{1}{(2 \pi)^{m}} \int_{0}^{2 \pi} \cdots \int_{0}^{2 \pi} \mathscr{P}^{\nu}\left(\Phi_{(i n)}(\theta, U), k^{\alpha}(J) \Phi_{(i n) \theta^{\alpha}}(\theta, U), \ldots\right) d^{m} \theta,
$$

we obtain that the system

$$
J^{v}(X)=\zeta^{v}(J(X), U(X))
$$

is satisfied by the solution $J^{v}(X) \equiv U^{v}(X)$ according to the definition of the parameters $U^{v}$. Since we suppose that the system (3.62) is of the generic form, we will assume that (locally) this is the only solution and put $J^{v}(X)=U^{v}(X)$ in the zero order of $\epsilon$.

After that we can resolve the system (3.60) by the iterations taking on the initial step $U^{v}(X)=J^{v}(X)$. The substitution of (3.56) into (3.60) under the condition of the non-singularity of matrix $\left.\left\|\partial \zeta^{\nu}(J, U) / \partial U^{\mu}\right\|\right|_{U=J}$ sequentially define the functions $u_{(k)}^{v}$. So we obtain the relations (3.56) and (3.57).

We can take also the values of $J^{v}(X), \theta_{0}^{* \alpha}(X), \theta_{0}^{\alpha}\left(X_{0}\right)$, and $G_{\left[U[\varphi], \theta_{0}[\varphi]\right]}^{i}(\theta, X)$ with the restrictions (3.55) and also

$$
\begin{gathered}
\frac{1}{(2 \pi)^{m}} \int_{0}^{2 \pi} \cdots \int_{0}^{2 \pi} \tilde{\kappa}_{(q)[U[\varphi](X)]}\left(\theta+\theta_{0}^{*}(X)+\theta_{0}\left(X_{0}\right)+\frac{1}{\epsilon} \int_{X_{0}}^{X} k\left(J\left(X^{\prime}\right)\right) d X^{\prime}\right) \\
\times G_{\left[U[\varphi], \theta_{0}[\varphi]\right]}^{i}(\theta, X) d^{m} \theta \equiv 0
\end{gathered}
$$

as the coordinates in the vicinity of $\mathcal{M}^{\prime}$.

We define the Poisson bracket on the space of functions $\varphi(\theta, X)$ by the formula

$$
\begin{aligned}
\left\{\varphi^{i}(\theta, X), \varphi^{j}\left(\theta^{\prime}, Y\right)\right\}= & \sum_{k \geq 0} B_{k}^{i j}\left(\varphi, \epsilon \varphi_{X}, \ldots\right) \epsilon^{k} \delta^{(k)}(X-Y) \delta\left(\theta-\theta^{\prime}\right) \\
& +\frac{1}{\epsilon} \delta\left(\theta-\theta^{\prime}\right) \sum_{k \geq 0} e_{k} S_{(k)}^{i}\left(\varphi, \epsilon \varphi_{X}, \ldots\right) v(X-Y) S_{(k)}^{j}\left(\varphi, \epsilon \varphi_{Y}, \ldots\right),
\end{aligned}
$$

which is just the rescaling of (1.1) multiplied by $\delta\left(\theta-\theta^{\prime}\right)$. We normalize the $\delta$-function $\delta\left(\theta-\theta^{\prime}\right)$ by $(2 \pi)^{m}$. 
The pairwise Poisson brackets of the constraints $G_{\left[U, \theta_{0}\right]}^{i}(\theta, X)$ on $\mathcal{M}^{\prime}$ can be written in the form

$$
\begin{aligned}
& \left.\left\{G^{i}(\theta, X), G^{j}\left(\theta^{\prime}, Y\right)\right\}\right|_{\mathcal{M}^{\prime}} \\
& \quad=\frac{1}{(2 \pi)^{2 m}} \int_{0}^{2 \pi} \cdots \int_{0}^{2 \pi} L_{k\left[U(X), \theta_{0}(X)\right]}^{i}(\theta, \tau) \\
& \quad \times L_{s\left[U(Y), \theta_{0}(Y)\right]}^{j}\left(\theta^{\prime}, \sigma\right) \times\left.\left\{\varphi^{k}(\tau, X), \varphi^{s}(\sigma, Y)\right\}\right|_{\mathcal{M}^{\prime}} d^{m} \tau d^{m} \sigma,
\end{aligned}
$$

(we can omit the Poisson brackets of $L_{k}^{i}$ and $L_{s}^{j}$ on $\mathcal{M}^{\prime}$ and also the brackets of the functionals $\theta_{0}^{\alpha}[\varphi]$ and $U^{\nu}[\varphi]$ from $\Phi_{(i n)}$ in (3.49) since they are multiplied by the convolutions of the corresponding $L$-operators with the "right eigenvectors" $\Phi_{(\text {in }) \theta^{\alpha}}$ and $\Phi_{(i n) U^{v}}$ which are zero on $\left.\mathcal{M}^{\prime}\right)$.

Brackets (3.65) evidently satisfy the orthogonality conditions

$$
\begin{aligned}
& \frac{1}{(2 \pi)^{m}} \int_{0}^{2 \pi} \cdots \int_{0}^{2 \pi} \tilde{\kappa}_{(q) i\left[U\left[J, \theta_{0}^{*}\right](X)\right]}\left(\theta+\theta_{0}^{*}(X)+\theta_{0}\left(X_{0}\right)+\frac{1}{\epsilon} \int_{X_{0}}^{X} k\left(J\left(X^{\prime}\right)\right) d X^{\prime}\right) \\
& \times\left.\left\{G^{i}(\theta, X), G^{j}\left(\theta^{\prime}, Y\right)\right\}\right|_{\mathcal{M}^{\prime}} d^{m} \theta=0, \\
&\left.\frac{1}{(2 \pi)^{m}} \int_{0}^{2 \pi} \cdots \int_{0}^{2 \pi}\left\{G^{i}(\theta, X), G^{j}\left(\theta^{\prime}, Y\right)\right\}\right|_{\mathcal{M}^{\prime}} \\
& \times \tilde{\kappa}_{(q) j\left[U\left[J, \theta_{0}^{*}\right](Y)\right]}\left(\theta+\theta_{0}^{*}(Y)+\theta_{0}\left(X_{0}\right)+\frac{1}{\epsilon} \int_{X_{0}}^{Y} k\left(J\left(Y^{\prime}\right)\right) d Y^{\prime}\right) d^{m} \theta^{\prime}=0
\end{aligned}
$$

for $q=1, \ldots, N+m$ in the coordinates $J(X), \theta_{0}^{*}(X)$, and $\theta_{0}\left(X_{0}\right)$ on the submanifold $\mu^{\prime}$.

We note that every derivative with respect to $X$ or $Y$ appears in the bracket (3.64) with the multiplier $\epsilon$ but being applied to the functions

$$
\varphi^{i}(\theta, X)=\Phi_{(i n)}^{i}\left(\theta+\theta_{0}^{*}(X)+\theta_{0}\left(X_{0}\right)+\frac{1}{\epsilon} \int_{X_{0}}^{X} k\left(J\left(X^{\prime}\right)\right) d X^{\prime}, U\left[J, \theta_{0}^{*}\right](X)\right)
$$

on $\mathcal{M}^{\prime}$ contains the nonzero at $\epsilon \rightarrow 0$ term $k^{\alpha}(J) \partial / \partial \theta^{\alpha}$. We formulate the statement about the structure of the bracket (3.64) on $\mathcal{M}^{\prime}$ in the coordinates $J(X), \theta_{0}^{*}(X)$, and $\theta_{0}\left(X_{0}\right)$.

LEMMA 3.6. The pairwise Poisson brackets of constraints $G_{\left[U, \theta_{0}\right]}^{i}(\theta, X)$ have no singular terms at $\epsilon \rightarrow 0$ and have no nonlocal terms in the zero order of $\epsilon\left(\epsilon^{0}\right)$ on $\mathcal{M}^{\prime}$ at any fixed coordinates $J^{v}(X), \theta_{0}^{* \alpha}(X)$, and $\theta_{0}^{\alpha}\left(X_{0}\right)$ (such that $U(X)=U\left[J, \theta_{0}^{*}\right](X)$, $\left.\theta_{0}(X)=\theta_{0}\left[J, \theta_{0}^{*}, \theta_{0}\left(X_{0}\right)\right](X)\right)$.

PRoof. The first statement is evident for the local part of bracket (3.64) since any differentiation with respect to $X$ in it appears with the multiplier $\epsilon$ and has the regular form at $\epsilon \rightarrow 0, k^{\alpha}(J(X)) \partial / \partial \theta^{\alpha}+O(\epsilon)$ being applied to the functions of the form (3.68). So we should check only the nonlocal part of (3.64) which contains the multiplier $\epsilon^{-1}$ in it. But according to the relation (3.47) and also (3.56), we have that the terms arising on both sides of $\nu(X-Y)$ (the convolutions of $\hat{L}$ with $S_{(k)}\left(\Phi, k^{\alpha} \Phi_{\theta^{\alpha}}, \ldots\right)$ ) are of order of $\epsilon$ on the $\mathcal{M}^{\prime}$ in the coordinates $J^{v}(X)$ and $\theta_{0}^{* \alpha}(X)$. So we obtain that all the nonlocal 
part of (3.65) is of order of $\epsilon$ on $\mathcal{M}^{\prime}$ at any fixed coordinates $J^{\nu}(X), \theta_{0}^{* \alpha}(X)$, and $\theta_{0}^{\alpha}\left(X_{0}\right)$. Lemma 3.6 is proved.

We formulate the last "regularity" property of the submanifold $\mathcal{M}^{\prime}$ with respect to the Poisson structure (3.64).

We consider, in the coordinates $J^{v}(X), \theta_{0}^{* \alpha}(X)$, and $\theta_{0}^{\alpha}\left(X_{0}\right)$ on $\mathcal{M}^{\prime}$, the linear nonhomogeneous system on the functions

$$
f_{j\left[J, \theta_{0}^{*}\right]}\left(\theta^{\prime}+\theta_{0}^{*}(Y)+\theta_{0}\left(X_{0}\right)+\frac{1}{\epsilon} \int_{X_{0}}^{Y} k\left(J\left(Y^{\prime}\right)\right) d Y^{\prime}, Y, \epsilon\right)
$$

having the form

$$
\begin{aligned}
& \left.\frac{1}{(2 \pi)^{m}} \int_{0}^{2 \pi} \cdots \int_{0}^{2 \pi}\left\{G_{\left[U[\varphi], \theta_{0}[\varphi]\right]}^{i}(\theta, X), G_{\left[U[\varphi], \theta_{0}[\varphi]\right]}^{j}\left(\theta^{\prime}, Y\right)\right\}\right|_{\mathcal{M}^{\prime}} \\
& \quad \times f_{j}\left(\theta^{\prime}+\theta_{0}^{*}(Y)+\theta_{0}\left(X_{0}\right)+\frac{1}{\epsilon} \int_{X_{0}}^{Y} k\left(J\left(Y^{\prime}\right)\right) d Y^{\prime}, Y, \epsilon\right) d^{m} \theta^{\prime} d Y \\
& =\left.\left\{G_{\left[U[\varphi], \theta_{0}[\varphi]\right]}^{i}(\theta, X), F[\varphi](\epsilon)\right\}\right|_{\mathcal{M}^{\prime}},
\end{aligned}
$$

where $F[\varphi](\epsilon)$ is the functional defined in the vicinity of $\boldsymbol{M}^{\prime}$.

After all differentiations, with respect to $X$, we can omit the term $\theta_{0}^{*}(X)+\theta_{0}\left(X_{0}\right)+$ $(1 / \epsilon) \int_{X_{0}}^{X} k^{\alpha}\left(J\left(X^{\prime}\right)\right) d X^{\prime}$ which occurs in all functions depending on $\theta$ and $X$ in (3.70), and then consider the system (3.70) at the zero order of $\epsilon$.

From Lemma 3.6, we have that at the zero order of $\epsilon$, the brackets $\left\{G^{i}(\theta, X)\right.$, $\left.G^{j}\left(\theta^{\prime}, Y\right)\right\}$ on $\mathcal{M}^{\prime}$ do not contain the nonlocal terms depending on $v(X-Y)$. For the derivatives, with respect to $X$, which arise with the multiplier $\epsilon$ from the local terms of $\left.\left\{\varphi^{k}(\tau, X), \varphi^{s}(\sigma, Y)\right\}\right|_{\mathcal{M}^{\prime}}$, we should take in the zero order of $\epsilon$ only the main part $k^{\alpha}(J) \partial / \partial \theta^{\alpha}$. So in the zero order of $\epsilon$, we obtain from (3.70) just the linear systems of the integral-differential equations with respect to $\theta$ and $\theta^{\prime}$ on the functions $f_{j}\left(\theta^{\prime}, X\right)$ independent at different $X$. We have also that the right-hand side of (3.70) satisfies at any $X$ and $\epsilon$ the compatibility conditions (3.66) (recall that $U^{v}\left[J, \theta_{0}^{*}\right]$ are the asymptotic series at $\epsilon \rightarrow 0$ ).

(IV) So we require that the system (3.70) is resolvable on $\mathcal{M}^{\prime}$ for any $F[\varphi](\epsilon)$ in the class of $2 \pi$-periodic with respect to all $\theta^{\alpha}$ functions, and its solutions can be represented in the form of regular at $\epsilon \rightarrow 0$ asymptotic series

$$
f_{j\left[J, \theta_{0}^{*}\right]}(\theta, Y, \epsilon)=\sum_{n \geq 0} \epsilon^{k} f_{j\left[J, \theta_{0}^{*}\right]}^{(k)}(\theta, Y)
$$

for regular at $\epsilon \rightarrow 0$ right-hand sides of (3.70).

Condition (IV) is responsible for the Dirac restriction of the bracket (3.64) on the submanifold $\mathcal{M}^{\prime}$.

We prove the statement which is very important for our averaging procedure. 
LEMMA 3.7. The Poisson brackets of the functionals $\theta_{0}^{* \alpha}(X)$ with $J^{v}(Y)$ are of order of $\epsilon$ at $\epsilon \rightarrow 0$ on the $\mathcal{M}^{\prime}$ at any fixed coordinates $J^{v}(X) \theta_{0}^{* \alpha}(X)$ and $\theta_{0}^{\alpha}\left(X_{0}\right)$

$$
\left.\left\{\theta_{0}^{* \alpha}(X), J^{\nu}(Y)\right\}\right|_{\mathcal{M}^{\prime}}=O(\epsilon), \quad \epsilon \longrightarrow 0
$$

Proof. First, we note that the Poisson brackets of $\varphi^{i}(\theta, X)$, with the functionals $J^{v}(Y)$, can be written in the form

$$
\begin{aligned}
& \left\{\varphi^{i}(\theta, X), J^{v}(Y)\right\} \\
& =\sum_{k \geq 0} C_{k}^{i v}\left(\varphi(\theta, X), \epsilon \varphi_{X}(\theta, X), \ldots\right) \epsilon^{k} \delta^{(k)}(X-Y) \\
& \quad+\sum_{k \geq 0} e_{k} S_{(k)}^{i}\left(\varphi(\theta, X), \epsilon \varphi_{X}(\theta, X), \ldots\right) v(X-Y)\left(F_{(k)}^{v}\left(\varphi(\theta, Y), \epsilon \varphi_{Y}(\theta, Y), \ldots\right)\right)_{Y},
\end{aligned}
$$

for some $C_{k}^{i v}\left(\varphi, \epsilon \varphi_{X}, \ldots\right)$ and $F_{(k)}^{v}\left(\varphi, \epsilon \varphi_{Y}, \ldots\right)$ (we have integrated with respect to $\theta^{\prime}$ ).

So the flow generated by the functional $\int q(Y) J^{v}(Y) d Y$ (where $q(Y)$ has a compact support) can be written as

$$
\begin{aligned}
\varphi_{t}^{i}= & \sum_{k \geq 0} C_{k}^{i v}\left(\varphi, \epsilon \varphi_{X}, \ldots\right) \epsilon^{k} q_{k X}(X) \\
& +\sum_{k \geq 0} e_{k} S_{(k)}^{i}\left(\varphi, \epsilon \varphi_{X}, \ldots\right) \int v(X-Y) q(Y) \frac{d}{d Y} F_{(k)}^{v}\left(\varphi, \epsilon \varphi_{Y}, \ldots\right) d Y \\
= & \sum_{k \geq 0} C_{k}^{i v}\left(\varphi, \epsilon \varphi_{X}, \ldots\right) \epsilon^{k} q_{k X}(X) \\
& +\sum_{k \geq 0} e_{k} S_{(k)}^{i}\left(\varphi, \epsilon \varphi_{X}, \ldots\right) F_{(k)}^{v}\left(\varphi, \epsilon \varphi_{X}, \ldots\right) q(X) \\
& -\sum_{k \geq 0} e_{k} S_{(k)}^{i}\left(\varphi, \epsilon \varphi_{X}, \ldots\right) \int v(X-Y) F_{(k)}^{v}\left(\varphi, \epsilon \varphi_{Y}, \ldots\right) q_{Y}(Y) d Y .
\end{aligned}
$$

As can be easily seen, the local terms of (3.74) have the form

$$
q(X)\left[C_{0}^{i v}\left(\varphi, \epsilon \varphi_{X}, \ldots\right)+\sum_{k \geq 0} e_{k} S_{(k)}^{i}\left(\varphi, \epsilon \varphi_{X}, \ldots\right) F_{(k)}^{v}\left(\varphi, \epsilon \varphi_{X}, \ldots\right)\right]+O(\epsilon)
$$

where the term in the brackets is just the flow generated by the functional

$$
\frac{1}{(2 \pi)^{m}} \iint_{0}^{2 \pi} \cdots \int_{0}^{2 \pi} \mathscr{P}^{v}\left(\varphi, \epsilon \varphi_{X}, \ldots\right) d^{m} \theta d X
$$

In the nonlocal part of (3.74) (the last expression) we have the convolution of the "slow" functions $q_{Y}(Y)$ with the rapidly oscillating $F_{(k)}^{v}\left(\varphi, \epsilon \varphi_{Y}, \ldots\right)$ where $\varphi^{i}(\theta, Y)$ has the form (3.68). So in the leading order of $\epsilon$, we can neglect the dependence on $\theta$ of the last integral of (3.74) and take the averaged, with respect to $\theta$ values, $\left\langle F_{(k)}^{v}\right\rangle$ on $\mathcal{M}^{\prime}$ instead of the exact $F_{(k)}^{v}\left(\varphi, \epsilon \varphi_{Y}, \ldots\right)$ in the integral expression in (3.74). 
After that we obtain that the nonlocal term of (3.74) gives us in the zero order of $\epsilon$, the linear combination of the flows $S_{(k)}\left(\varphi, \epsilon \varphi_{X}, \ldots\right)$, considered on the functions

$$
\varphi^{i}(\theta, X)=\Phi_{(i n)}^{i}\left(\theta+\theta_{0}^{*}(X)+\theta_{0}\left(X_{0}\right)+\frac{1}{\epsilon} \int_{X_{0}}^{X} k\left(J\left(X^{\prime}\right)\right) d X^{\prime}, J(X)\right)
$$

at any fixed point $X$.

From the invariance of the submanifold $\mu$ with respect to the flows (3.27), we can conclude that the flow (3.74) being considered at the points of $\mathcal{M}^{\prime}$ with fixed coordinates $J(X), \theta_{0}^{*}(X), \theta_{0}\left(X_{0}\right)$ in the zero order of $\epsilon$, leaves $\mu^{\prime}$ invariant and generates on it the linear evolution of the initial phases

$$
\theta_{0}^{\alpha}(X)=\theta_{0}^{* \alpha}(X)+\theta_{0}^{\alpha}\left(X_{0}\right)+\frac{1}{\epsilon} \int_{X_{0}}^{X} k^{\alpha}\left(J\left(X^{\prime}\right)\right) d X^{\prime},
$$

with some frequencies $\Omega_{[q]}^{\alpha \nu}(X)$. We use the formula (3.56) for $U\left[J, \theta_{0}^{*}\right]$ and we can claim that the Poisson brackets of the functionals $\theta_{0}^{\alpha}(X)$ with $\int q(Y) J^{v}(Y) d Y$ at the points of $\mathcal{M}^{\prime}$ with fixed coordinates $J^{v}(X), \theta_{0}^{* \alpha}(X)$, and $\theta_{0}\left(X_{0}\right)$ have the form

$$
\left\{\theta_{0}^{\alpha}(X), \int q(Y) J^{v}(Y) d Y\right\}=\Omega_{[q]}^{\alpha v}\left[J, \theta_{0}^{*}\right](X)+O(\epsilon) .
$$

We prove the relation

$$
\left\{k^{\alpha}(J(X)), \int q(Y) J^{v}(Y) d Y\right\}=\epsilon \frac{d}{d X} \Omega_{[q]}^{\alpha v}\left[J, \theta_{0}^{*}\right](X)+O\left(\epsilon^{2}\right)
$$

at the points of $\mathcal{M}^{\prime}$ with the fixed values of $J^{\nu}(X), \theta_{0}^{* \alpha}(X)$, and $\theta_{0}^{\alpha}\left(X_{0}\right)$.

Using again the relation (3.56), we can write for (3.74) at the points of $\mathcal{M}^{\prime}$

$$
\begin{aligned}
\varphi_{t}^{i}= & \Omega_{[q]}^{\beta v}(X) \Phi_{(i n) \theta^{\beta}}^{i}\left(\theta+\theta_{0}^{*}(X)+\theta_{0}\left(X_{0}\right)+\frac{1}{\epsilon} \int_{X_{0}}^{X} k\left(J\left(X^{\prime}\right)\right) d X^{\prime}, U\left[J, \theta_{0}^{*}\right](X)\right) \\
& +\epsilon \eta^{i}\left(\theta+\theta_{0}^{*}(X)+\theta_{0}\left(X_{0}\right)+\frac{1}{\epsilon} \int_{X_{0}}^{X} k\left(J\left(X^{\prime}\right)\right) d X^{\prime},\left[J, \theta_{0}^{*}\right]\right),
\end{aligned}
$$

where $\left[J, \theta_{0}^{*}\right]$ means the regular at $\epsilon \rightarrow 0$ dependence on $J, J_{X}, \theta_{0 X}^{*}, \ldots$

We are interested in the evolution of the functionals

$$
J^{\mu}(X)=\frac{1}{(2 \pi)^{m}} \int_{0}^{2 \pi} \cdots \int_{0}^{2 \pi} \mathscr{P}^{\mu}\left(\varphi, \epsilon \varphi_{X}, \ldots\right) d^{m} \theta .
$$

We have

$$
\begin{aligned}
\frac{d}{d t} J^{\mu}(X) & =\frac{1}{(2 \pi)^{m}} \int_{0}^{2 \pi} \cdots \int_{0}^{2 \pi}\left(\frac{\partial \mathscr{P}^{\mu}}{\partial \varphi^{i}} \varphi_{t}^{i}+\frac{\partial \mathscr{P}^{\mu}}{\partial \varphi_{X}^{i}} \varphi_{t X}^{i}+\frac{\partial \mathscr{P}^{\mu}}{\partial \varphi_{X X}^{i}} \varphi_{t X X}^{i}+\ldots\right) d^{m} \theta \\
& =\frac{1}{(2 \pi)^{m}} \int_{0}^{2 \pi} \cdots \int_{0}^{2 \pi}\left(\prod_{i(0)}^{\mu} \varphi_{t}^{i}+\prod_{i(1)}^{\mu} \epsilon \varphi_{t X}^{i}+\prod_{i(2)}^{\mu} \epsilon^{2} \varphi_{t X X}^{i}+\cdots\right) d^{m} \theta
\end{aligned}
$$

where the densities $\prod_{i(k)}^{\mu}$ were introduced in (3.35). 
It is easy to see that (3.81) does not change $J^{\mu}(X)$ at the zero order of $\epsilon$ and we can also state that the terms of order of $\epsilon$ in (3.81) (i.e., $\left.\epsilon \eta^{i}(\theta+\cdots, X)\right)$ are unessential for the evolution of $k(J(X))$ on $\mathcal{M}^{\prime}$ at the order of $\epsilon$. Indeed, their contribution to the evolution of $J^{\mu}(X)$ in the order of $\epsilon$ is

$$
\epsilon \frac{1}{(2 \pi)^{m}} \int_{0}^{2 \pi} \cdots \int_{0}^{2 \pi}\left(\prod_{i(0)}^{\mu} \eta^{i}+\prod_{i(1)}^{\mu} \epsilon \eta_{X}^{i}+\prod_{i(2)}^{\mu} \epsilon^{2} \eta_{X X}^{i}+\cdots\right) d^{m} \theta
$$

where we should take only the main term $k^{\gamma}(J(X)) \partial / \partial \theta^{\gamma}$ for the derivatives $\epsilon \partial / \partial X$ in the formula (3.84). After the integration by parts, we have for this contribution

$$
\epsilon \frac{1}{(2 \pi)^{m}} \int_{0}^{2 \pi} \cdots \int_{0}^{2 \pi}\left(\prod_{i(0)}^{\mu}-k^{\gamma} \frac{\partial}{\partial \theta^{\gamma}} \prod_{i(1)}^{\mu}+\cdots\right) \eta^{i}(\theta+\cdots, X) d^{m} \theta .
$$

But after the substitution of the main part of $\varphi^{i}(\theta, X)$

$$
\Phi_{(i n)}^{i}\left(\theta+\theta_{0}^{*}(X)+\theta_{0}\left(X_{0}\right)+\frac{1}{\epsilon} \int_{X_{0}}^{X} k\left(J\left(X^{\prime}\right)\right) d X^{\prime}, J(X)\right)
$$

(according to (3.56)) into the densities $\prod_{i(k)}^{\mu}\left(\varphi, \epsilon \varphi_{X}, \ldots\right)$, we obtain in the leading order of $\epsilon$ the convolution of $\eta(\theta, X)$ with the variational derivative of the functional $\bar{I}^{\mu}$ introduced in (3.25) with respect to $\varphi(\theta, X)$. Our statement follows from Lemma 3.1 which claims that the variational derivatives of the functionals $k^{\alpha}(\bar{I}[\varphi])$ are identically zero on the space of $m$-phase solutions of (2.7).

Consider the first term of (3.81). We have that the evolution of $J^{\mu}(X)$ which is responsible for the evolution of $k(J)$ is given by the expression

$$
\begin{aligned}
\frac{d}{d t} J^{\mu}(X)= & \Omega_{[q]}^{\beta v}(X) \\
& \times \frac{1}{(2 \pi)^{m}} \int_{0}^{2 \pi} \cdots \int_{0}^{2 \pi}\left(\frac{\partial \Phi^{\mu}}{\partial \varphi^{i}} \Phi_{(i n) \theta^{\beta}}^{i}\left(\theta+\mathbf{s}(X, \epsilon), U\left[J, \theta_{0}^{*}\right](X)\right)\right. \\
& \left.+\frac{\partial \Phi^{\mu}}{\partial \varphi_{X}^{i}} \frac{\partial}{\partial X} \Phi_{(i n) \theta^{\beta}}^{i}\left(\theta+\mathbf{s}(X, \epsilon), U\left[J, \theta_{0}^{*}\right](X)\right)+\ldots\right) d^{m} \theta \\
+ & \left.\epsilon\left(\Omega_{[q]}^{\beta v}(X)\right)_{X} \frac{1}{(2 \pi)^{m}} \int_{0}^{2 \pi} \cdots \int_{0}^{2 \pi}\left(\frac{\partial \mathscr{P}^{\mu}}{\partial \varphi_{X}^{i}}+2 \frac{\partial \mathscr{P}^{\mu}}{\partial \varphi_{X X}^{i}} \frac{\partial}{\partial X}+3 \frac{\partial \mathscr{P}^{\mu}}{\partial \varphi_{X X X}^{i}} \frac{\partial^{2}}{\partial X^{2}}+\ldots\right)\right) \\
& \times \Phi_{(i n) \theta^{\beta}}^{i}\left(\theta+\mathbf{s}(X, \epsilon), U\left[J, \theta_{0}^{*}\right](X)\right) d^{m} \theta+O\left(\epsilon^{2}\right),
\end{aligned}
$$

where $\mathbf{s}(X, \epsilon) \equiv \theta_{0}^{*}(X)+\theta_{0}\left(X_{0}\right)+(1 / \epsilon) \int_{X_{0}}^{X} k\left(J\left(X^{\prime}\right)\right) d X^{\prime}$.

The first term here after the substitution of exact $\varphi^{i}$ in the form

$$
\varphi^{i}(\theta, X)=\Phi_{(i n) \theta^{\beta}}^{i}\left(\theta+\mathbf{s}(X, \epsilon), U\left[J, \theta_{0}^{*}\right](X)\right),
$$


on the $\mathcal{M}^{\prime}$ as can be easily seen is just

$$
\Omega_{[q]}^{\beta v}(X) \frac{1}{(2 \pi)^{m}} \int_{0}^{2 \pi} \cdots \int_{0}^{2 \pi} \frac{\partial}{\partial \theta^{\beta}} \mathscr{P}^{\mu}\left(\Phi_{(i n)}(\ldots), \Phi_{(i n) X}(\ldots), \ldots\right) d^{m} \theta \equiv 0
$$

while the second term on $\mathcal{M}^{\prime}$ in the leading order of $\epsilon$ is equal to

$$
\begin{aligned}
\epsilon\left(\Omega_{[q]}^{\beta v}(X)\right)_{X} \frac{1}{(2 \pi)^{m}} \int_{0}^{2 \pi} \cdots \int_{0}^{2 \pi} \sum_{p \geq 1} p \prod_{i(p)}^{\mu}\left(\Phi_{(i n)}(\theta+\mathbf{s}(X, \epsilon), J(X)),\right. \\
\left.k^{\gamma}(J(X)) \frac{\partial}{\partial \theta^{\gamma}} \Phi_{(i n)}(\theta+\mathbf{s}(X, \epsilon), J(X)), \ldots\right) \\
\times k^{\alpha_{1}}(J(X)) \cdots k^{\alpha_{p-1}}(J(X)) \Phi_{(i n) \theta^{\beta} \theta^{\alpha_{1}} \cdots \theta^{\alpha_{p-1}}}^{i} \\
\times(\theta+\mathbf{s}(X, \epsilon), J(X), \ldots) d^{m} \theta,
\end{aligned}
$$

which coincides with the integral expression from (3.36) in Lemma 3.4. So from Lemma 3.4 we have that the summation of (3.90) with $\partial k^{\alpha} / \partial J^{\mu}$ is equal to $\epsilon\left(\Omega_{[q]}^{\beta v}(X)\right)_{X}$ $\delta_{\beta}^{\alpha}$ and we obtain that

$$
\frac{\partial}{\partial t} k^{\alpha}(J)=\epsilon \frac{\partial}{\partial X} \Omega_{[q]}^{\alpha \nu}(X)+O\left(\epsilon^{2}\right)
$$

that is, the relation (3.80).

Now using (3.79) and (3.80), we can write that

$$
\begin{aligned}
& \left\{\theta_{0}^{* \alpha}(X), \int q(Y) J^{v}(Y) d Y\right\} \\
& \quad=\left\{\theta_{0}^{\alpha}(X)-\theta_{0}^{\alpha}\left(X_{0}\right)-\frac{1}{\epsilon} \int_{X_{0}}^{X} k^{\alpha}\left(J\left(X^{\prime}\right)\right) d X^{\prime}, \int q(Y) J^{v} d Y\right\}=O(\epsilon),
\end{aligned}
$$

for any $q(Y)$ in our coordinates on $\mathcal{M}^{\prime}$.

So we have

$$
\left.\left\{\theta_{0}^{* \alpha}(X), J^{\nu}(Y)\right\}\right|_{\mathcal{M}^{\prime}}=O(\epsilon)
$$

at any fixed coordinates $J^{v}(X), \theta_{0}^{* \alpha}(X)$, and $\theta_{0}^{\alpha}\left(X_{0}\right)$. Lemma 3.7 is proved.

4. Averaging procedure. We formulate the averaging procedure of the Poisson bracket (1.1) on the family of $m$-phase solutions of (2.7) under the conditions of "regularity" formulated above.

THEOREM 4.1. Consider the Poisson brackets (1.1) and the local system (2.7) generated by the local Hamiltonian function

$$
H=\int \mathscr{P}_{H}\left(\varphi, \varphi_{x}, \ldots\right) d x
$$

which has $N(\geq 2 m)$-parametric full family of $m$-phase solutions modulo $m$ initial phase shifts $\theta_{0}^{\alpha}$. 
Assume that there exist $N$ commutative local translation invariant integrals

$$
I^{v}=\int \mathscr{P}^{v}\left(\varphi, \varphi_{x}, \ldots\right) d x, \quad\left\{I^{v}, H\right\}=0,\left\{I^{v}, I^{\mu}\right\}=0,
$$

which generate local flows according to the Poisson bracket (1.1) and the averaged densities of which can be taken as parameters $U^{v}$ on the space of $m$-phase solutions of (2.7) (the conditions (A), (B), and (C)).

Then under the "regularity" conditions (I), (II), (III), and (IV) for the space of m-phase solutions of (2.7), we can construct a Poisson bracket of Ferapontov type (2.6) for the "slow" parameters $U^{v}(X)$ which coincide with the averaged densities $\left\langle\mathscr{P}^{v}\right\rangle(X)$ by the following procedure: we calculate the pairwise Poisson brackets of $\mathscr{P}^{v}\left(\varphi, \varphi_{x}, \ldots\right)$ in the form

$$
\begin{aligned}
& \left\{\mathscr{P}^{v}\left(\varphi, \varphi_{x}, \ldots\right), \mathscr{P}^{\mu}\left(\varphi, \varphi_{y}, \ldots\right)\right\} \\
& =\sum_{k \geq 0} A_{k}^{v \mu}\left(\varphi, \varphi_{x}, \ldots\right) \delta^{(k)}(x-y) \\
& \quad+\sum_{k \geq 0} e_{k}\left(F_{(k)}^{v}\left(\varphi, \varphi_{x}, \ldots\right)\right)_{x} v(x-y)\left(F_{(k)}^{\mu}\left(\varphi, \varphi_{y}, \ldots\right)\right)_{y},
\end{aligned}
$$

(there are finite numbers of terms in both sums). (The total derivatives of the functions $F_{(k)}^{v}$ and $F_{(k)}^{\mu}$ with respect to $x$ and $y$ arise as a corollary of the fact that both $I^{v}$ and $I^{\mu}$ generate the local flows (3.22).) From the commutativity of the set $\left\{I^{v}\right\}$, it follows also that

$$
\begin{aligned}
& A_{0}^{v \mu}\left(\varphi, \varphi_{x}, \ldots\right) \\
& \quad+\sum_{k \geq 0} e_{k}\left(F_{(k)}^{v}\left(\varphi, \varphi_{x}, \ldots\right)\right)_{x} F_{(k)}^{\mu}\left(\varphi, \varphi_{x}, \ldots\right) \equiv \partial_{x} Q^{v \mu}\left(\varphi, \varphi_{x}, \ldots\right)
\end{aligned}
$$

for some functions $Q^{\nu \mu}\left(\varphi, \varphi_{x}, \ldots\right)$.

Then for the "slow" coordinates $U^{v}(X)=\left\langle\mathscr{P}^{v}\right\rangle(X)$, we can define the Poisson bracket by the formula

$$
\begin{aligned}
\left\{U^{v}(X), U^{\mu}(Y)\right\}= & {\left[\left\langle A_{1}^{v \mu}\right\rangle(X)+\sum_{k \geq 0} e_{k}\left(\left\langle F_{(k)}^{v} F_{(k)}^{\mu}\right\rangle-\left\langle F_{(k)}^{v}\right\rangle\left\langle F_{(k)}^{\mu}\right\rangle\right)(X)\right] \delta^{\prime}(X-Y) } \\
& +\left[\frac{\partial\left\langle Q^{v \mu}\right\rangle(X)}{\partial X}-\sum_{k \geq 0} e_{k} \frac{\partial\left\langle F_{(k)}^{v}\right\rangle(X)}{\partial X}\left\langle F_{(k)}^{\mu}\right\rangle(X)\right] \delta(X-Y) \\
& +\sum_{k \geq 0} e_{k} \frac{\partial\left\langle F_{(k)}^{v}\right\rangle(X)}{\partial X} v(X-Y) \frac{\partial\left\langle F_{(k)}^{\mu}\right\rangle(Y)}{\partial Y},
\end{aligned}
$$

where the averaged values are the functions of $U(X)$ and $U(Y)$ at the corresponding $X$ and $Y$.

Bracket (4.5) satisfies the Jacobi identity, and is invariant with respect to the choice of the set $\left\{I^{1}, \ldots, I^{N}\right\}$ satisfying $(A),(B)$, and $(C)$ if it is possible to choose such integrals in different ways, that is, if $U^{v}=\left\langle\mathscr{P}^{v}\right\rangle, \tilde{U}^{v}=\left\langle\tilde{\mathscr{P}}^{v}\right\rangle$ and $\left\{U^{v}(X), U^{\mu}(Y)\right\},\left\{\tilde{U}^{v}(X), \tilde{U}^{\mu}(Y)\right\}^{\prime}$ are the brackets (4.5) constructed with the sets $\left\{I^{v}\right\}$ and $\left\{\tilde{I}^{v}\right\}$, respectively, then

$$
\left\{\tilde{U}^{v}(X), \tilde{U}^{\mu}(Y)\right\}^{\prime} \equiv \frac{\partial \tilde{U}^{v}}{\partial U^{\lambda}}(X)\left\{U^{\lambda}(X), U^{\sigma}(Y)\right\} \frac{\partial \tilde{U}^{\mu}}{\partial U^{\sigma}}(Y) .
$$


Proof. The most difficult part is to prove the Jacobi identity for the bracket (4.5). For this we use the Dirac restriction of the Poisson bracket (3.64) on the submanifold $\mathcal{M}^{\prime}$ with the coordinates $J^{v}(X), \theta_{0}^{* \alpha}(X)$, and $\theta_{0}^{\alpha}\left(X_{0}\right)$ on it. According to the Dirac restriction procedure we should find for $J^{v}(X), \theta_{0}^{* \alpha}(X)$, and $\theta_{0}^{\alpha}\left(X_{0}\right)$ the additions of type

$$
\begin{aligned}
V^{v}(X) & =\frac{1}{(2 \pi)^{m}} \int_{0}^{2 \pi} \cdots \int_{0}^{2 \pi} \int v_{j}^{v}\left[J, \theta_{0}^{* \alpha}, \theta_{0}\left(X_{0}\right)\right]\left(X, \theta^{\prime}, Z\right) G^{j}\left(\theta^{\prime}, Z\right) d^{m} \theta^{\prime} d Z \\
W^{\alpha}(X) & =\frac{1}{(2 \pi)^{m}} \int_{0}^{2 \pi} \cdots \int_{0}^{2 \pi} \int w_{j}^{\alpha}\left[J, \theta_{0}^{* \alpha}, \theta_{0}\left(X_{0}\right)\right]\left(X, \theta^{\prime}, Z\right) G^{j}\left(\theta^{\prime}, Z\right) d^{m} \theta^{\prime} d Z \\
O^{\alpha} & =\frac{1}{(2 \pi)^{m}} \int_{0}^{2 \pi} \cdots \int_{0}^{2 \pi} \int o_{j}^{\alpha}\left[J, \theta_{0}^{* \alpha}, \theta_{0}\left(X_{0}\right)\right]\left(\theta^{\prime}, Z\right) G^{j}\left(\theta^{\prime}, Z\right) d^{m} \theta^{\prime} d Z
\end{aligned}
$$

such that the fluxes generated view (3.64) by the "functionals" $J^{v}(X)+V^{v}(X)$, $\theta_{0}^{* \alpha}(X)+W^{\alpha}(X)$, and $\theta_{0}^{\alpha}\left(X_{0}\right)+O^{\alpha}$ leave $\mathcal{M}^{\prime}$ invariant, that is,

$$
\begin{aligned}
& \left.\frac{1}{(2 \pi)^{m}} \int_{0}^{2 \pi} \cdots \int_{0}^{2 \pi}\left\{G^{i}(\theta, Y), G^{j}\left(\theta^{\prime}, Z\right)\right\}\right|_{\mathcal{M}^{\prime}} \times v_{j}^{v}\left(X, \theta^{\prime}, Z\right) d^{m} \theta^{\prime} d Z \\
& \quad=-\left.\left\{G^{i}(\theta, Y), J^{v}(X)\right\}\right|_{\mathcal{M}^{\prime}}, \\
& \left.\frac{1}{(2 \pi)^{m}} \int_{0}^{2 \pi} \cdots \int_{0}^{2 \pi}\left\{G^{i}(\theta, Y), G^{j}\left(\theta^{\prime}, Z\right)\right\}\right|_{\mathcal{M}^{\prime}} \times w_{j}^{\alpha}\left(X, \theta^{\prime}, Z\right) d^{m} \theta^{\prime} d Z \\
& \quad=-\left.\left\{G^{i}(\theta, Y), \theta_{0}^{* \alpha}(X)\right\}\right|_{\mathcal{M}^{\prime}}, \\
& \left.\frac{1}{(2 \pi)^{m}} \int_{0}^{2 \pi} \cdots \int_{0}^{2 \pi}\left\{G^{i}(\theta, Y), G^{j}\left(\theta^{\prime}, Z\right)\right\}\right|_{\mathcal{M}^{\prime}} \times o_{j}^{\alpha}\left(\theta^{\prime}, Z\right) d^{m} \theta^{\prime} d Z \\
& \quad=-\left.\left\{G^{i}(\theta, Y), \theta_{0}^{\alpha}\left(X_{0}\right)\right\}\right|_{\mathcal{M}^{\prime}},
\end{aligned}
$$

and put after that for the Dirac restriction on $\mathcal{M}^{\prime}$

$$
\begin{aligned}
& \left\{J^{v}(X), J^{\mu}(Y)\right\}_{D} \\
& \quad=\left.\left\{J^{v}(X)+V^{v}(X), J^{\mu}(Y)+V^{\mu}(Y)\right\}\right|_{\mathcal{M}^{\prime}} \\
& \quad=\left.\left\{J^{v}(X), J^{\mu}(Y)\right\}\right|_{\mathcal{M}^{\prime}}-\frac{1}{(2 \pi)^{2 m}} \int_{0}^{2 \pi} \cdots \int_{0}^{2 \pi} v_{i}^{v}(X, \theta, Z) \times v_{j}^{\mu}\left(Y, \theta^{\prime}, Z^{\prime}\right) \\
& \quad \times\left.\left\{G^{i}(\theta, Z), G^{j}\left(\theta^{\prime}, Z^{\prime}\right)\right\}\right|_{\mathcal{M}^{\prime}} d^{m} \theta d^{m} \theta^{\prime} d Z d Z^{\prime},
\end{aligned}
$$

and by the same way

$$
\begin{aligned}
\left\{J^{v}(X), \theta_{0}^{* \alpha}(Y)\right\}_{D}= & \left.\left\{J^{v}(X), \theta_{0}^{* \alpha}(Y)\right\}\right|_{\mathcal{M}^{\prime}} \\
& -\frac{1}{(2 \pi)^{2 m}} \int_{0}^{2 \pi} \cdots \int_{0}^{2 \pi} v_{i}^{v}(X, \theta, Z) \times w_{j}^{\alpha}\left(Y, \theta^{\prime}, Z^{\prime}\right) \\
& \quad \times\left.\left\{G^{i}(\theta, Z), G^{j}\left(\theta^{\prime}, Z^{\prime}\right)\right\}\right|_{\mathcal{M}^{\prime}} d^{m} \theta d^{m} \theta^{\prime} d Z d Z^{\prime}
\end{aligned}
$$




$$
\begin{aligned}
\left\{\theta_{0}^{* \alpha}(X), \theta_{0}^{* \beta}(Y)\right\}_{D}= & \left.\left\{\theta_{0}^{* \alpha}(X), \theta_{0}^{* \beta}(Y)\right\}\right|_{\mathcal{M}^{\prime}} \\
& -\frac{1}{(2 \pi)^{2 m}} \int_{0}^{2 \pi} \cdots \int_{0}^{2 \pi} w_{j}^{\alpha}(X, \theta, Z) \times w_{j}^{\beta}\left(Y, \theta^{\prime}, Z^{\prime}\right) \\
& \times\left.\left\{G^{i}(\theta, Z), G^{j}\left(\theta^{\prime}, Z^{\prime}\right)\right\}\right|_{\mathcal{M}^{\prime}} d^{m} \theta d^{m} \theta^{\prime} d Z d Z^{\prime},
\end{aligned}
$$

and so on.

After the calculation of the brackets in (4.8) and (4.9) and the substitution of $\varphi(\theta, X)$ in the form (3.68) we obtain the regular at $\epsilon \rightarrow 0$ systems for the functions $\bar{v}_{j}^{v}(X, \theta, Z, \epsilon)$, $\bar{w}_{j}^{\alpha}(X, \theta, Z, \epsilon)$, and $\bar{o}_{j}^{\alpha}(\theta, Z, \epsilon)$ such that

$$
\begin{aligned}
v_{j}^{v}(X, \theta, Z, \epsilon) & =\bar{v}_{j}^{v}\left(X, \theta+\theta_{0}^{*}(Z)+\theta_{0}\left(X_{0}\right)+\frac{1}{\epsilon} \int_{X_{0}}^{Z} k\left(J\left(Z^{\prime}\right)\right) d Z^{\prime}, \epsilon\right), \\
w_{j}^{\alpha}(X, \theta, Z, \epsilon) & =\bar{w}_{j}^{\alpha}\left(X, \theta+\theta_{0}^{*}(Z)+\theta_{0}\left(X_{0}\right)+\frac{1}{\epsilon} \int_{X_{0}}^{Z} k\left(J\left(Z^{\prime}\right)\right) d Z^{\prime}, \epsilon\right), \\
o_{j}^{\alpha}(\theta, Z, \epsilon) & =\bar{o}_{j}^{\alpha}\left(\theta+\theta_{0}^{*}(Z)+\theta_{0}\left(X_{0}\right)+\frac{1}{\epsilon} \int_{X_{0}}^{Z} k\left(J\left(Z^{\prime}\right)\right) d Z^{\prime}, \epsilon\right),
\end{aligned}
$$

which coincide with the system (3.70).

From the arguments analogous to those used in Lemma 3.6 and the fact that the flows generated by the functionals $J^{v}(X)$ leave invariant the submanifold $\mathcal{M}^{\prime}$ at the zero order of $\epsilon$ in the coordinates $J(X), \theta_{0}^{*}(X)$, and $\theta_{0}\left(X_{0}\right)$ we have also that the right-hand sides of these systems are regular at $\epsilon \rightarrow 0$ in these coordinates.

So according to (IV) we can find the functions $\bar{v}_{j}^{v}, \bar{w}_{j}^{\alpha}$, and $\bar{o}_{j}^{\alpha}$ in the form of regular at $\epsilon \rightarrow 0$ asymptotic series. (The functions $v_{j}^{v}(X, \theta, Z, \epsilon), w_{j}^{\alpha}(X, \theta, Z, \epsilon)$, and $o_{j}^{\alpha}(\theta, Z, \epsilon)$ are not uniquely defined but it can be shown that it does not influence on the Dirac restriction of the bracket (3.64) on $\mathcal{M}^{\prime}$ according to the formulas (4.10) and (4.11).)

Besides that, as was mentioned above, the flows (3.74) generated by the functionals $\int q(X) J^{\mu}(X) d X$ on the functions of the form (3.68) leave invariant the submanifold $\mathcal{M}^{\prime}$ at the zero order of $\epsilon$ and generate the linear evolution of the initial phases. So we can conclude that the right-hand side of the linear system (4.8) contains no zero powers of $\epsilon$ and we should start the expansion for $\bar{v}_{i}^{v}(X, \theta, Z, \epsilon)$ from the first power.

Now we have

$$
\begin{aligned}
\bar{v}_{j}^{v}\left[J, \theta_{0}^{*}, \theta_{0}\left(X_{0}\right)\right](X, \theta, Z, \epsilon) & =\sum_{k \geq 1} \epsilon^{k} \bar{v}_{j(k)}^{v}\left[J, \theta_{0}^{*}, \theta_{0}\left(X_{0}\right)\right](X, \theta, Z), \\
\bar{w}_{j}^{\alpha}\left[J, \theta_{0}^{*}, \theta_{0}\left(X_{0}\right)\right](X, \theta, Z, \epsilon) & =\sum_{k \geq 0} \epsilon^{k} \bar{w}_{j(k)}^{\alpha}\left[J, \theta_{0}^{*}, \theta_{0}\left(X_{0}\right)\right](X, \theta, Z), \\
\bar{o}_{j}^{\alpha}\left[J, \theta_{0}^{*}, \theta_{0}\left(X_{0}\right)\right](\theta, Z, \epsilon) & =\sum_{k \geq 0} \epsilon^{k} \bar{o}_{j(k)}^{\alpha}\left[J, \theta_{0}^{*}, \theta_{0}\left(X_{0}\right)\right](\theta, Z) .
\end{aligned}
$$

According to the relations above and Lemma 3.6, we can see that the corrections to the values $\left.\left\{J^{v}(X), J^{\mu}(Y)\right\}\right|_{\mathcal{M}^{\prime}}$ and $\left.\left\{\theta_{0}^{* \alpha}(X), J^{\mu}(Y)\right\}\right|_{\mathcal{M}^{\prime}}$, due to the Dirac restriction on $\mathcal{M}^{\prime}$, are of order of $O\left(\epsilon^{2}\right)$ and $O(\epsilon)$, respectively.

According to the relation (3.56), we can also substitute the values $J^{v}(X)$ instead of $U^{v}\left[J, \theta_{0}^{*}\right](X)$ in the leading order of $\epsilon$ as the arguments of the averaged functions on $M^{\prime}$. 
We calculate the values $\left.\left\{J^{\nu}(X), J^{\mu}(Y)\right\}\right|_{\mathcal{M}^{\prime}}$. Let the Poisson brackets

$$
\left.\left\{\int q^{v}(X) J^{v}(X) d X, \int q^{\mu}(Y) J^{\mu}(Y) d Y\right\}\right|_{\mathcal{H}^{\prime}}
$$

for the arbitrary smooth $q^{v}(X)$ and $q^{\mu}(Y)$ with compact supports. For the Poisson brackets of the densities $\mathscr{P}^{v}\left(\varphi, \epsilon \varphi_{X}, \ldots\right)$, according to (3.64), we have the expression

$$
\begin{aligned}
& \left\{\mathscr{P}^{v}(\theta, X), \mathscr{P}^{\mu}\left(\theta^{\prime}, Y\right)\right\} \\
& =\sum_{k \geq 0} A_{k}^{v \mu}\left(\varphi, \epsilon \varphi_{X}, \ldots\right) \epsilon^{k} \delta^{(k)}(X-Y) \delta\left(\theta-\theta^{\prime}\right) \\
& \quad+\epsilon \delta\left(\theta-\theta^{\prime}\right) \sum_{k \geq 0} e_{k}\left(F_{(k)}^{v}\left(\varphi, \epsilon \varphi_{X}, \ldots\right)\right)_{X} v(X-Y)\left(F_{(k)}^{\mu}\left(\varphi, \epsilon \varphi_{Y}, \ldots\right)\right)_{Y},
\end{aligned}
$$

such that

$$
\begin{aligned}
& \left\{J^{v}(X), J^{\mu}(Y)\right\} \\
& =\sum_{k \geq 0} \epsilon^{k} \frac{1}{(2 \pi)^{m}} \int_{0}^{2 \pi} \cdots \int_{0}^{2 \pi} A_{k}^{v \mu}\left(\varphi(\theta, X), \epsilon \varphi_{X}(\theta, X), \ldots\right) d^{m} \theta \delta^{(k)}(X-Y) \\
& +\epsilon \sum_{k \geq 0} e_{k} \frac{1}{(2 \pi)^{m}} \int_{0}^{2 \pi} \cdots \int_{0}^{2 \pi}\left(F_{(k)}^{v}\left(\varphi(\theta, X), \epsilon \varphi_{X}(\theta, X), \ldots\right)\right)_{X} \\
& \times v(X-Y) \times\left(F_{(k)}^{\mu}\left(\varphi(\theta, Y), \epsilon \varphi_{Y}(\theta, Y), \ldots\right)\right)_{Y} .
\end{aligned}
$$

We should substitute the functions $\varphi^{i}(\theta, X), \varphi^{i}(\theta, Y)$ on $\mu^{\prime}$ in the form

$$
\begin{aligned}
& \Phi_{(\text {in })}^{i}\left(\theta+\theta_{0}^{*}(X)+\theta_{0}\left(X_{0}\right)+\frac{1}{\epsilon} \int_{X_{0}}^{X} k\left(J\left(X^{\prime}\right)\right) d X^{\prime}, U\left[J, \theta_{0}^{*}\right](X)\right), \\
& \Phi_{(\text {in })}^{i}\left(\theta+\theta_{0}^{*}(Y)+\theta_{0}\left(X_{0}\right)+\frac{1}{\epsilon} \int_{X_{0}}^{Y} k\left(J\left(Y^{\prime}\right)\right) d Y^{\prime}, U\left[J, \theta_{0}^{*}\right](Y)\right),
\end{aligned}
$$

respectively.

It is easy to see that the local part of (4.16) gives us the expression

$$
\begin{gathered}
\frac{1}{(2 \pi)^{m}} \int_{0}^{2 \pi} \cdots \int_{0}^{2 \pi} d^{m} \theta \times A_{0}^{v \mu}\left(\Phi_{(i n)}^{i}\left(\theta+s(X), U\left[J, \theta_{0}^{*}\right](X)\right),\right. \\
\left.\epsilon \frac{\partial}{\partial X} \Phi_{(i n)}^{i}\left(\theta+s(X), U\left[J, \theta_{0}^{*}\right](X)\right), \ldots\right) \\
\times \delta(X-Y)+\epsilon\left\langle A_{1}^{v \mu}\right\rangle(J(X)) \delta^{\prime}(X-Y)+O\left(\epsilon^{2}\right),
\end{gathered}
$$

in the coordinates $J(X), \theta_{0}^{*}(X)$, and $\theta_{0}\left(X_{0}\right)$ on $\mathcal{M}^{\prime}$ where $s(X) \equiv \theta_{0}^{*}(X)+\theta_{0}\left(X_{0}\right)+$ $(1 / \epsilon) \int_{X_{0}}^{X} k\left(J\left(X^{\prime}\right)\right) d X^{\prime}$. 
Here we used only the main part of $(4.17) \Phi_{(i n)}^{i}(\theta+s(X), J(X))$ in the second term of (4.19) and replaced $U^{v}(X)$ by $J^{v}(X)$ according to (3.56) in the arguments of the averaged functions modulo the higher orders of $\epsilon$.

In the nonlocal part of (4.16) we have for (4.14) the following equalities:

$$
\begin{aligned}
& \iint d X d Y \frac{1}{(2 \pi)^{m}} \int_{0}^{2 \pi} \cdots \int_{0}^{2 \pi} \frac{1}{\epsilon} \sum_{k \geq 0} e_{k} \in q^{v}(X) \frac{\partial F_{(k)}^{v}\left(\Phi_{(i n)}(\theta+s(X), U(X)), \ldots\right)}{\partial X} \times v(X-Y) \\
& \times \epsilon q^{\mu}(Y) \frac{\partial F_{(k)}^{\mu}\left(\Phi_{(i n)}(\theta+s(Y), U(Y)), \ldots\right)}{\partial Y} d^{m} \theta \\
& =\iint d X d Y \frac{1}{(2 \pi)^{m}} \int_{0}^{2 \pi} \cdots \int_{0}^{2 \pi} \sum_{k \geq 0} e_{k} \frac{\partial^{2}\left[q^{v}(X) v(X-Y) q^{\mu}(Y)\right]}{\partial X \partial Y} \\
& \times F_{(k)}^{v}\left(\Phi_{(i n)}(\theta+s(X), U(X)), \ldots\right) \\
& \times F_{(k)}^{\mu}\left(\Phi_{(i n)}(\theta+s(Y), U(Y)), \ldots\right) d^{m} \theta \\
& =\iint d X d Y \frac{1}{(2 \pi)^{m}} \int_{0}^{2 \pi} \cdots \int_{0}^{2 \pi} \sum_{k \geq 0} e_{k} \epsilon\left[q_{X}^{\nu}(X) \nu(X-Y) q_{Y}^{\mu}(Y)\right. \\
& +\left(q^{v}(X) q_{Y}^{\mu}(Y)-q_{X}^{v}(X) q^{\mu}(Y)\right) \delta(X-Y) \\
& \left.-q^{v}(X) q^{\mu}(Y) \delta^{\prime}(X-Y)\right] \\
& \times F_{(k)}^{v}\left(\Phi_{(i n)}(\theta+s(X), U(X)), \ldots\right) \\
& \times F_{(k)}^{\mu}\left(\Phi_{(i n)}(\theta+s(Y), U(Y)), \ldots\right) d^{m} \theta \\
& =\iint d X d Y \frac{1}{(2 \pi)^{m}} \int_{0}^{2 \pi} \cdots \int_{0}^{2 \pi} \sum_{k \geq 0} e_{k} \epsilon q_{X}^{v}(X) v(X-Y) q_{Y}^{\mu}(Y) \\
& \times F_{(k)}^{v}\left(\Phi_{(i n)}(\theta+s(X), U(X)), \ldots\right) \\
& \times F_{(k)}^{\mu}\left(\Phi_{(i n)}(\theta+s(Y), U(Y)), \ldots\right) d^{m} \theta \\
& +\epsilon \sum_{k \geq 0} e_{k} \int\left(q^{v}(X) q_{X}^{\mu}(X)-q_{X}^{v}(X) q^{\mu}(X)\right)\left\langle F_{(k)}^{v} F_{(k)}^{\mu}\right\rangle(J(X)) d X \\
& -\epsilon \sum_{k \geq 0} e_{k} \int q^{v}(X) q_{X}^{\mu}(X)\left\langle F_{(k)}^{v} F_{(k)}^{\mu}\right\rangle(J(X)) d X \\
& -\epsilon \int q^{v}(X) q^{\mu}(X) \frac{1}{(2 \pi)^{m}} \int_{0}^{2 \pi} \cdots \int_{0}^{2 \pi} \sum_{k \geq 0} e_{k} F_{(k)}^{v}\left(\Phi_{(i n)}(\theta+s(X), U(X)), \ldots\right) \\
& \times \frac{\partial}{\partial X} F_{(k)}^{\mu}\left(\Phi_{(i n)}(\theta+s(X), U(X)), \ldots\right) \\
& \times d^{m} \theta d X+O\left(\epsilon^{2}\right) \text {, }
\end{aligned}
$$

where we used the integration by parts for the generalized functions. 
We can see now that in the first term of the expression above in both regions $X>Y$ and $X<Y$ we have the convolution with respect to $X$ and $Y$, of the "slow" functions $q_{X}^{v}(X) q_{Y}^{\mu}(Y)$ with the rapidly oscillating expression

$$
\left\langle F_{(k)}^{v}\left(\Phi_{(i n)}(\theta+s(X, \epsilon), J(X)), \ldots\right) F_{(k)}^{\mu}\left(\Phi_{(i n)}(\theta+s(Y, \epsilon), J(Y)), \ldots\right)\right\rangle,
$$

in the main order of $\epsilon$. Here $\langle\cdots\rangle$ means the averaging with respect to phases $\theta^{\alpha}$. So as the small $\Delta X$ and $\Delta Y$ lead in the main order of $\epsilon$, to the changes of phase difference equal to $(1 / \epsilon) k^{\alpha}(J(X)) \Delta X+O\left((\Delta X)^{2}\right)$ and $(1 / \epsilon) k^{\alpha}(J(Y)) \Delta Y+O\left((\Delta Y)^{2}\right)$ it is not very difficult to see that in the sense of "generalized" limit (i.e., in the sense of the convolutions with the "slow" functions of $X$ and $Y$ ) we can replace this oscillating expression just by its mean value

$$
\sum_{k \geq 0} e_{k}\left\langle F_{(k)}^{v}\right\rangle(J(X))\left\langle F_{(k)}^{\mu}\right\rangle(J(Y))
$$

where $\langle\cdots\rangle$ means the averaging on the space of $m$-phase solutions.

As for the last term of (4.20), we recall that its sum with the expression arising from the first term of the local part in (4.19)

$$
\begin{array}{r}
\int d X q^{v}(X) q^{\mu}(X) \frac{1}{(2 \pi)^{m}} \int_{0}^{2 \pi} \cdots \int_{0}^{2 \pi} d^{m} \theta\left[A_{0}^{v \mu}\left(\Phi_{(i n)}(\theta+s(X), U(X)), \ldots\right)\right. \\
-\sum_{k \geq 0} e_{k} F_{(k)}^{v}\left(\Phi_{(i n)}(\theta+s(X), U(X)), \ldots\right) \\
\left.\times \in \frac{\partial}{\partial X} F_{(k)}^{\mu}\left(\Phi_{(i n)}(\theta+s(X), U(X)), \ldots\right)\right]
\end{array}
$$

is equal, according to (4.4), to

$$
\epsilon \int\left(\frac{\partial\left\langle Q^{v \mu}\right\rangle(J(X))}{\partial X}-\sum_{k \geq 0} e_{k} \frac{\partial\left\langle F_{(k)}^{v} F_{(k)}^{\mu}\right\rangle(J(X))}{\partial X}\right) q^{v}(X) q^{\mu}(X) d X,
$$

in the leading order of $\epsilon$.

So we can write

$$
\begin{aligned}
& \left.\left\{\int q^{v}(X) J^{v}(X) d X, \int q^{\mu}(Y) J^{\mu}(Y) d Y\right\}\right|_{\mathcal{M}^{\prime}} \\
& =\epsilon \int\left[q^{v}(X) q_{X}^{\mu}(X)\left\langle A_{1}^{v \mu}\right\rangle(J(X))-q_{X}^{v}(X) q^{\mu}(X) \sum_{k \geq 0} e_{k}\left\langle F_{(k)}^{v} F_{(k)}^{\mu}\right\rangle(J(X))\right. \\
& \left.\quad+q^{v}(X) q^{\mu}(X) \partial_{X}\left(\left\langle Q^{v \mu}\right\rangle(J(X))-\sum_{k \geq 0} e_{k}\left\langle F_{(k)}^{v} F_{(k)}^{\mu}\right\rangle(J(X))\right)\right] d X \\
& \quad+\epsilon \iint \sum_{k \geq 0} e_{k} q_{X}^{v}(X)\left\langle F_{(k)}^{v}\right\rangle(J(X)) v(X-Y)\left\langle F_{(k)}^{\mu}\right\rangle(J(Y)) q_{Y}^{\mu}(Y) d X d Y+O\left(\epsilon^{2}\right) .
\end{aligned}
$$


After the integration by parts (in the sense of generalized functions) we can write this expression in the following "canonical" form:

$$
\begin{aligned}
& \left.\left\{\int q^{v}(X) J^{v}(X) d X, \int q^{\mu}(Y) J^{\mu}(Y) d Y\right\}\right|_{\mathcal{M}^{\prime}} \\
& =\epsilon \int\left(\left\langle A_{1}^{v \mu}\right\rangle(J(X))+\sum_{k \geq 0} e_{k}\left(\left\langle F_{(k)}^{v} F_{(k)}^{\mu}\right\rangle(J(X))-\left\langle F_{(k)}^{v}\right\rangle(J(X))\left\langle F_{(k)}^{\mu}\right\rangle(J(X))\right)\right) \\
& \quad \times q^{v}(X) q_{X}^{\mu}(X) d X \\
& \quad+\epsilon \int\left(\frac{\partial\left\langle Q^{v \mu}\right\rangle(J(X))}{\partial X}-\sum_{k \geq 0} e_{k} \frac{\partial\left\langle F_{(k)}^{v}\right\rangle(J(X))}{\partial X}\left\langle F_{(k)}^{\mu}\right\rangle(J(X))\right) q^{v}(X) q^{\mu}(X) d X \\
& \quad+\epsilon \iint \sum_{k \geq 0} e_{k} q^{v}(X) \frac{\partial\left\langle F_{(k)}^{v}\right\rangle(J(X))}{\partial X} v(X-Y) \frac{\partial\left\langle F_{(k)}^{\mu}\right\rangle(J(Y))}{\partial Y} q^{\mu}(Y) d X d Y+O\left(\epsilon^{2}\right),
\end{aligned}
$$

which corresponds to the bracket

$$
\begin{aligned}
\left.\left\{J^{v}(X), J^{\mu}(Y)\right\}\right|_{\mathcal{M}^{\prime}} & \\
= & \epsilon\left(\left\langle A_{1}^{v \mu}\right\rangle(J(X))+\sum_{k \geq 0} e_{k}\left(\left\langle F_{(k)}^{v} F_{(k)}^{\mu}\right\rangle-\left\langle F_{(k)}^{v}\right\rangle\left\langle F_{(k)}^{\mu}\right\rangle\right)(J(X))\right) \delta^{\prime}(X-Y) \\
& +\epsilon\left(\frac{\partial\left\langle Q^{v \mu}\right\rangle(J(X))}{\partial X}-\sum_{k \geq 0} e_{k} \frac{\partial\left\langle F_{(k)}^{v}\right\rangle(J(X))}{\partial X}\left\langle F_{(k)}^{\mu}\right\rangle(J(X))\right) \delta(X-Y) \\
& +\epsilon \sum_{k \geq 0} e_{k} \frac{\partial\left\langle F_{(k)}^{v}\right\rangle(J(X))}{\partial X} \nu(X-Y) \frac{\partial\left\langle F_{(k)}^{\mu}\right\rangle(J(Y))}{\partial Y}+O\left(\epsilon^{2}\right)
\end{aligned}
$$

for the functionals $J^{v}(X)$.

So according to Lemma 3.7 and the remarks above, we obtain for the Dirac restriction on $M^{\prime}$

$$
\left\{\theta_{0}^{* \alpha}(X), J^{\mu}(Y)\right\}_{D}=O(\epsilon),
$$

and the relations (4.27) for the brackets $\left\{J^{\nu}(X), J^{\mu}(Y)\right\}_{D}$ in the coordinates $J(X)$, $\theta_{0}^{*}(X)$, and $\theta_{0}\left(X_{0}\right)$.

It is evident also that the Dirac brackets $\left\{J^{v}(X), J^{\mu}(Y)\right\}_{D}$ on $\mathcal{M}^{\prime}$ do not depend at any order of $\epsilon$, on the common initial phase $\theta_{0}\left(X_{0}\right)$ because of the invariance of $J^{v}(X)$, the bracket (3.64), and the submanifold $\mu^{\prime}$ with respect to the common shifts of $\theta^{\alpha}$.

The dependence of $\left\{J^{v}(X), J^{\mu}(Y)\right\}_{D}$ on $J(X), J_{X}(X), \theta_{0 X}^{*}(X), \ldots$ is regular at $\epsilon \rightarrow 0$ and as can be easily seen from (4.27), we have not any dependence of $\theta_{0}^{*}$ in the first order of $\epsilon$.

So it is easy to see now that the Jacobi identities for the bracket $\{\cdots, \cdots\}_{D}$ on $\mathcal{M}^{\prime}$ with coordinates $J(X), \theta_{0}^{*}(X)$, and $\theta_{0}\left(X_{0}\right)$ written for the fields $J^{\nu}(X), J^{\mu}(Y)$, and 
$J^{\lambda}(Z)$ at the order of $\epsilon^{2}$ coincide with the corresponding Jacobi identities for the bracket (4.5).

So we proved the Jacobi identity for the bracket (4.5).

The skew-symmetry of the bracket (4.5) is just the trivial corollary of the skewsymmetry of (3.64).

We prove the invariance of the bracket (4.5) with respect to the choice of the integrals $I^{v}$. The proof is just the same as in the local case and we just reproduce it here.

Under the condition (IV) (which is the "generic" requirement) we have the unique restriction of the Poisson bracket (3.64) on $\mathcal{M}^{\prime}$ with the coordinates $J(X), \theta_{0}^{*}(X), \theta_{0}\left(X_{0}\right)$ in the form of formal series at $\epsilon \rightarrow 0$.

So the two restrictions of (3.64) obtained in the coordinates

$$
\begin{aligned}
& \left(J^{v}(X), \theta_{0}^{* \alpha}(X), \theta_{0}^{\alpha}\left(X_{0}\right)\right), \\
& \left(\tilde{J}^{v}(X), \tilde{\theta}_{0}^{* \alpha}(X), \theta_{0}^{\alpha}\left(X_{0}\right)\right),
\end{aligned}
$$

corresponding to the sets $\left\{I^{v}\right\}$ and $\left\{\tilde{I}^{v}\right\}$ (satisfying (A), (B), and (C)), respectively, should transform one into another after the corresponding transformation of coordinates

$$
\begin{aligned}
& \tilde{J}^{v}(X)=\tilde{j}_{(0)}^{v}(J(X))+\sum_{k \geq 1} \epsilon^{k} \tilde{j}_{(k)}^{v}\left[J, \theta_{0}^{*}\right](X), \\
& \tilde{\theta}_{0}^{* \alpha}(X)=\tilde{\theta}_{0}^{* \alpha}\left[J, \theta_{0}^{*}, \epsilon\right](X)
\end{aligned}
$$

on $\mathcal{M}^{\prime}$.

We note that the leading term of (4.27) coinciding with the bracket (4.5), transforms according to the transformation

$$
\tilde{J}^{v}(X)=\tilde{j}_{(0)}^{v}(J(X))
$$

which corresponds to the substitution $\tilde{U}^{v}(X)=\tilde{U}^{v}(U(X))$ on $\mu^{\prime}$ view the relation (3.56). So we obtain the second part of the theorem. Theorem 4.1 is proved.

REMARK 4.2. From Theorem 4.1 it also follows, in particular, that the procedure (4.5) is insensitive to the addition of the total derivatives with respect to $x$ to the densities $\mathscr{P}^{v}\left(\varphi, \varphi_{x}, \ldots\right)$. This fact however can also be obtained from the elementary consideration of the definition of bracket (4.5).

THEOREM 4.3. The Hamiltonian functions

$$
\begin{aligned}
\bar{H}^{v} & =\int U^{v}(X) d X, \\
\bar{H} & =\int\left\langle\mathscr{P}_{H}\right\rangle(U(X)) d X,
\end{aligned}
$$

generate view (4.5) the local-commuting flows which give us the Whitham equations for the systems (3.22) and (2.7), respectively. 
Proof. It is easy to check by the direct substitution that any of $\bar{H}^{\mu}$ generates the "conservative" form

$$
U_{T}^{v}=\partial_{X}\left\langle Q^{v \mu}\right\rangle(U)
$$

of Whitham's system for the corresponding flow (3.22). It is easy to see also that this flow conserves any of $\bar{H}^{v}$ so that any $\bar{H}^{v}$ and $\bar{H}^{\mu}$ commute view the bracket (4.5). The same property for the Hamiltonian function $\bar{H}$ (and also for the integral of the averaged density of any local integral $I$ commuting with $H$ and $I^{v}$ and generating the local flow view (1.1)) can be obtained from the invariance of (4.5) with respect to the set $\left\{I^{v}\right\}$ since we can use the Hamiltonian function $H$ in the form of (2.2) as the one of the integrals instead of any of $I^{v}$. Theorem 4.3 is proved.

We can also see that the functionals $\bar{H}^{v}$ give us the conservation laws for our Whitham system.

From Theorem 2.1, it follows also that the flows

$$
U_{T}^{v}=\partial_{X}\left\langle F_{(k)}^{v}\right\rangle(U)
$$

commute with all the local flows generated by local functionals $\int h(U) d X$ in the Hamiltonian structure (4.5) and it can also be seen that they give us Whitham's equations for the corresponding flows (2.8).

It can be easily seen also that the described procedure can be applied by the same way to the brackets (2.1) written also in the "irreducible" form and not only in the "canonical" one.

ACKNowledgments. The author is grateful to B. A. Dubrovin, S. P. Novikov, V. L. Alekseev, O. I. Mokhov, M. V. Pavlov, and E. V. Ferapontov for fruitful discussions. The work was partially supported by International Association (grant INTAS 96-0770) and Russian Foundation for Basic Research (grant 97-01-00281).

\section{REFERENCES}

[1] V. L. Alekseev, On nonlocal Hamiltonian operators of hydrodynamic type that are associated with the Whitham equation, Uspekhi Mat. Nauk 50 (1995), no. 6(306), 165-166.

[2] V. L. Alekseev and M. V. Pavlov, Hamiltonian structures of the Whitham equations, Proceedings of the Conference on NLS, Chernogolovka, 1994.

[3] L. V. Bogdanov and E. V. Ferapontov, A nonlocal Hamiltonian formalism for semiHamiltonian systems of hydrodynamic type, Teoret. Mat. Fiz. 116 (1998), no. 1, 113-121.

[4] S. J. Dobrohotov and V. P. Maslov, Finite-zone almost periodic solutions in WKBapproximations, Current Problems in Mathematics, Vol. 15 (Russian), Akad. Nauk SSSR, Moscow, 1980, pp. 3-94.

[5] B. A. Dubrovin and S. P. Novikov, Hamiltonian formalism of one-dimensional systems of the hydrodynamic type and the Bogolyubov-Whitham averaging method, Soviet Math. Dokl. 27 (1983), 665-669, [translated from Dokl. Akad. Nauk SSSR 270 (1983), 781-785 ].

[6] _ Hydrodynamics of weakly deformed soliton lattices. Differential geometry and Hamiltonian theory, Uspekhi Mat. Nauk 44 (1989), no. 6(270), 29-98. 

ical Physics Reviews, Part 4, vol. 9, Harwood Academic Publishers GmbH, Yverdon, 1993.

[8] B. Enriquez, V. Rubtsov, and A. Orlov, Higher Hamiltonian structures (the $\mathrm{sl}_{2}$ case), Pis ma Zh. Èksper. Teoret. Fiz. 58 (1993), no. 8, 677-683.

[9] E. V. Ferapontov, Differential geometry of nonlocal Hamiltonian operators of hydrodynamic type, Funktsional. Anal. i Prilozhen. 25 (1991), no. 3, 37-49.

[10] - Nonlocal matrix Hamiltonian operators. Differential geometry and applications, Teoret. Mat. Fiz. 91 (1992), no. 3, 452-462.

[11] Restriction, in the sense of Dirac, of the Hamiltonian operator $\delta^{I J}(d / d x)$ to a surface of the Euclidean space with a plane normal connection, Funktsional. Anal. i Prilozhen. 26 (1992), no. 4, 83-86.

[12] On integrability of $3 \times 3$ semi-Hamiltonian hydrodynamic type systems $u_{t}^{i}=$ $v_{j}^{i}(u) u_{x}^{j}$ which do not possess Riemann invariants, Phys. D 63 (1993), no. 1-2, 50-70.

[13]___ On the matrix Hopf equation and integrable Hamiltonian systems of hydrodynamic type, which do not possess Riemann invariants, Phys. Lett. A 179 (1993), no. 6, 391397.

[14]__ Several conjectures and results in the theory of integrable Hamiltonian systems of hydrodynamic type, which do not possess Riemann invariants, Teoret. Mat. Fiz. 99 (1994), no. 2, 257-262.

[15] _ Dupin hypersurfaces and integrable Hamiltonian systems of hydrodynamic type, which do not possess Riemann invariants, Differential Geom. Appl. 5 (1995), no. 2, 121-152.

[16] _ Nonlocal Hamiltonian operators of hydrodynamic type: differential geometry and applications, Topics in topology and mathematical physics, Amer. Math. Soc. Transl. Ser. 2, vol. 170, American Mathematical Society, Rhode Island, 1995, pp. 3358.

[17] H. Flaschka, M. G. Forest, and D. W. McLaughlin, Multiphase averaging and the inverse spectral solution of the Korteweg-de Vries equation, Comm. Pure Appl. Math. 33 (1980), no. 6, 739-784.

[18] N. I. Grinberg, On Poisson brackets of hydrodynamic type with a degenerate metric, Russian Math. Surveys 40 (1985), no. 4, 231-232.

[19] I. M. Krichever, The averaging method for two-dimensional "integrable" equations, Funktsional. Anal. i Prilozhen. 22 (1988), no. 3, 37-52, 96.

[20] J. C. Luke, A perturbation method for nonlinear dispersive wave problems, Proc. Roy. Soc. Ser. A 292 (1966), 403-412.

[21] A. Ya. Maltsev, Averaging of local field-theoretic Poisson brackets, Uspekhi Mat. Nauk 52 (1997), no. 2(314), 177-178.

[22] _ Averaging of Hamiltonian structures in a discrete variant of the Whitham method, Uspekhi Mat. Nauk 53 (1998), no. 1(319), 219-220.

[23] _ Inheritance of Hamiltonian structures in the Whitham averaging method, Izv. Ross. Akad. Nauk Ser. Mat. 63 (1999), no. 6, 117-146.

[24] A. Ya. Maltsev and S. P. Novikov, On the local systems Hamiltonian in the weakly non-local Poisson brackets, Phys. D 156 (2001), no. 1-2, 53-80.

[25] A. Ya. Maltsev and M. V. Pavlov, On Whitham's averaging method, Funktsional. Anal. i Prilozhen. 29 (1995), no. 1, 7-24.

[26] O. I. Mokhov and E. V. Ferapontov, Nonlocal Hamiltonian operators of hydrodynamic type that are connected with metrics of constant curvature, Uspekhi Mat. Nauk 45 (1990), no. 3(273), 191-192.

[27] S. P. Novikov, A periodic problem for the Korteweg-de Vries equation. I, Funkcional. Anal. i Priložen. 8 (1974), no. 3, 54-66. 
[28] S. P. Novikov and A. Ya. Maltsev, Liouville form of averaged Poisson brackets, Uspekhi Mat. Nauk 48 (1993), no. 1(289), 155-156.

[29] M. V. Pavlov, Multi-Hamiltonian structures of the Whitham equations, Russian Acad. Sci. Dokl. Math. 50 (1995), no. 2, 220-223, [translated from Dokl. Akad. Nauk 338 (1994), 165-167 ].

[30] V. V.Sokolov, Hamiltonian property of the Krichever-Novikov equation, Soviet Math. Dokl. 30 (1984), no. 1, 44-46.

[31] S. P. Tsarëv, Poisson brackets and one-dimensional Hamiltonian systems of hydrodynamic type, Soviet Math. Dokl. 31 (1985), 488-491, [translated from Dokl. Akad. Nauk SSSR 282 (1985), 534-537 ].

[32] G. B. Whitham, Linear and Nonlinear Waves, Pure and Applied Mathematics, John Wiley \& Sons, New York, 1974.

ANDRei YA. MALTSEV: LANDAU INSTITUTE FOR THEORETICAL PHysics, 117940, Kosygina 2, MosCOW, RUSSIA

E-mail address: ma1tsev@itp.ac.ru

Current address: SISSA-ISAS, VIA BEIRUT 2-4, 34014 TRIESTE, ITALY

E-mail address: ma7tsev@sissa.it 


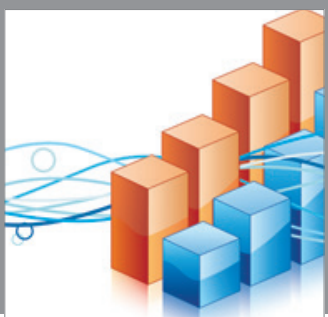

Advances in

Operations Research

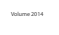

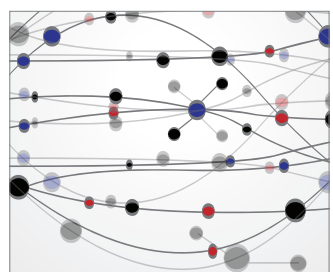

\section{The Scientific} World Journal
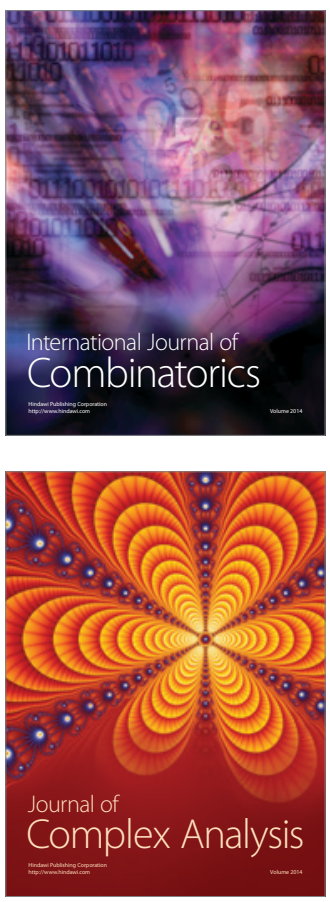

International Journal of

Mathematics and

Mathematical

Sciences
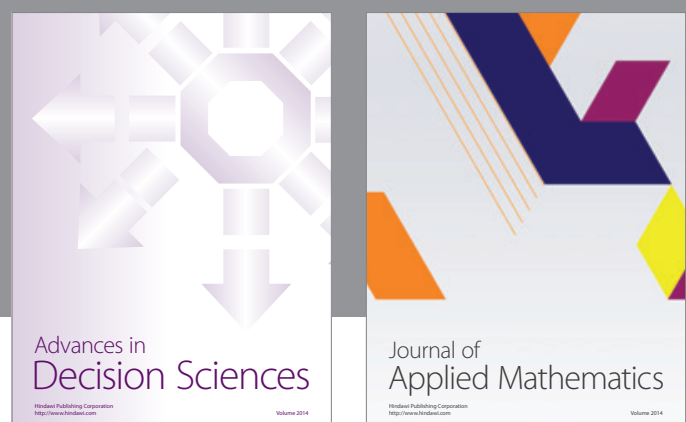

Journal of

Applied Mathematics
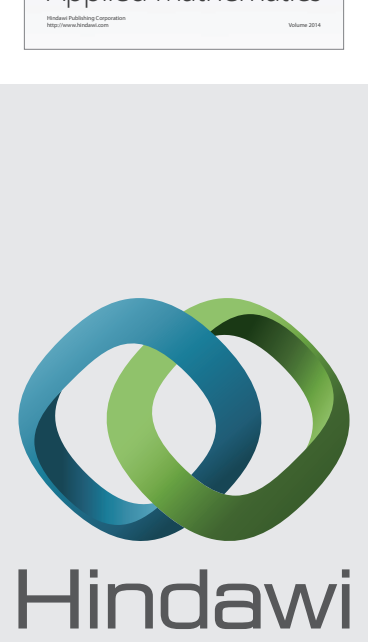

Submit your manuscripts at http://www.hindawi.com
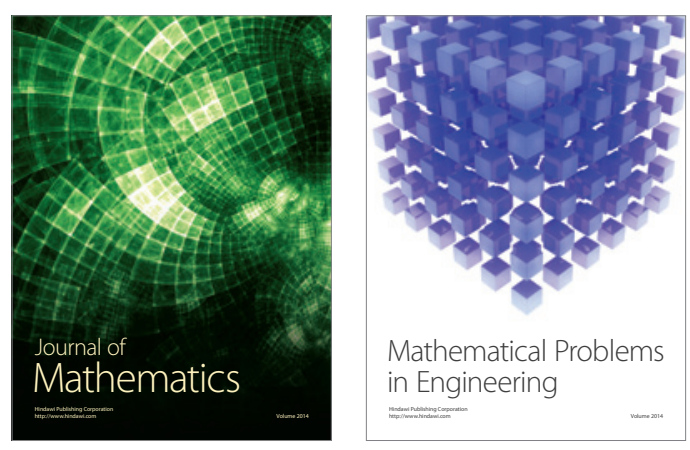

Mathematical Problems in Engineering
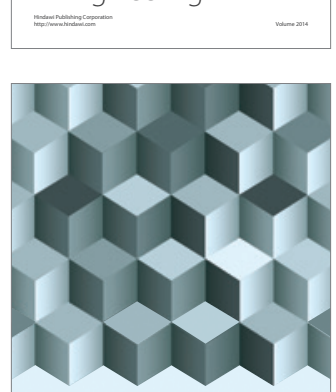

Journal of

Function Spaces
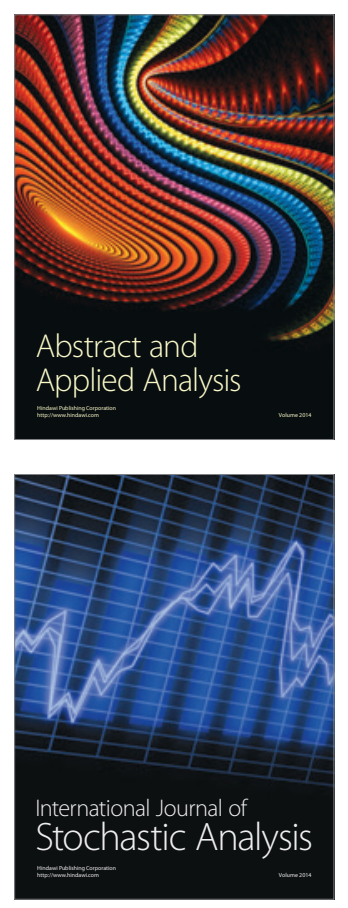

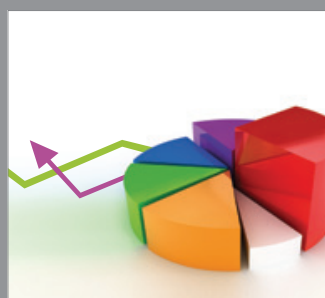

ournal of

Probability and Statistics

Promensencen
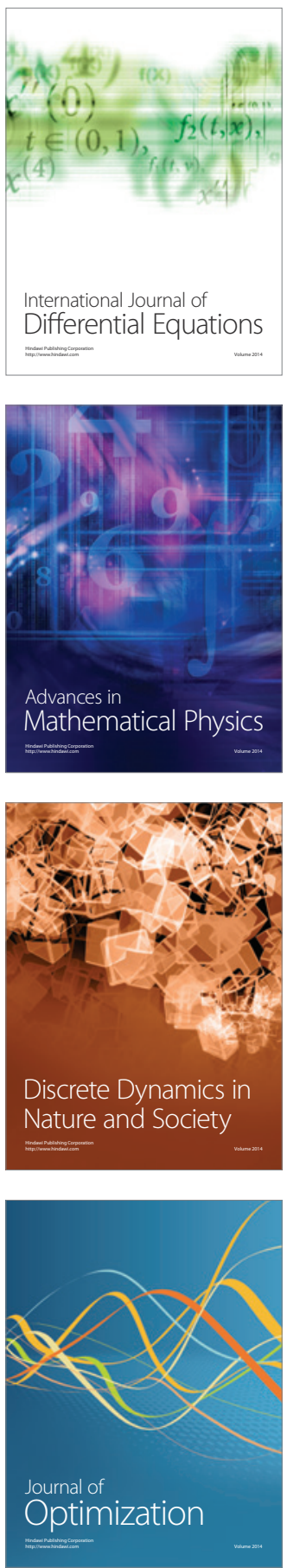